\title{
ON THE STANDARD POISSON STRUCTURE AND A FROBENIUS SPLITTING OF THE BASIC AFFINE SPACE
}

\author{
JUN PENG AND SHIZHUO YU
}

\begin{abstract}
The goal of this paper is to construct a Frobenius splitting on $G / U$ via the Poisson geometry of $\left(G / U, \pi_{G / U}\right)$, where $G$ is a simply-connected semi-simple algebraic group defined over an algebraically closed field of characteristic $p>3, U$ is the uniradical of a Borel subgroup of $G$ and $\pi_{G / U}$ is the standard Poisson structure on $G / U$. We first study the Poisson geometry of $\left(G / U, \pi_{G / U}\right)$. Then, we develop a general theory for Frobenius splittings on $\mathbb{T}$-Poisson varieties, where $\mathbb{T}$ is an algebraic torus. In particular, we prove that compatibly split subvarieties of Frobenius splittings constructed in this way must be $\mathbb{T}$-Poisson subvarieties. Lastly, we apply our general theory to construct a Frobenius splitting on $G / U$.
\end{abstract}

\section{INTRODUCTION AND STATEMENTS OF RESUltS}

1.1. Introduction. Let $G$ be a simply-connected semi-simple algebraic group over an algebraically closed field $k$ and $U$ the uniradical of a Borel subgroup of $G$. The quasi-affine variety $G / U$ is called the basic affine space. When $k=\mathbb{C}$, there exists the well-known standard Poisson structure $\pi_{\text {st }}$ on $G$, which has its roots in the theory of quantum groups (see [3] [8]). The standard Poisson structure $\pi_{\text {st }}$ projects to a well-defined Poisson structure $\pi_{G / U}$ called the standard Poisson structure on $G / U$.

Let $\mathbb{T}$ be a complex torus. A $\mathbb{T}$-Poisson manifold, defined in [16, Section 1.1], is a complex Poisson manifold $\left(X, \pi_{X}\right)$ with a $\mathbb{T}$-action preserving the Poisson structure $\pi_{X}$. A $\mathbb{T}$-Poisson manifold gives rise to a decomposition of $X$ into the union of $\mathbb{T}$-leaves of $\pi_{X}$, a term defined in [16, Section 2.2]. Every single $\mathbb{T}$-leaf in $\left(X, \pi_{X}\right)$ admits a non-zero anti-canonical section on $X$ called the Poisson $\mathbb{T}$-Pfaffian, a term suggested by A. Knutson and M. Yakimov (see [16, Section 1.1]). When $k=\mathbb{C}, G$ becomes a complex semi-simple Lie group. Let $T$ be a maximal torus of $G$ normalizing $U$. The left translation of $T$ on $G / U$ makes $\left(G / U, \pi_{G / U}\right)$ into a natural $T$-Poisson manifold. Although J.-H. Lu and V. Mouquin develop a general theory (see [16, Section 1.5]) to compute the $\mathbb{T}$-leaves of a class of $\mathbb{T}$-Poisson manifolds, the case $\left(G / U, \pi_{G / U}\right)$ is an exceptional example. Using the Bruhat decomposition on $G / U$ and some propositions about the so-called $\mathbb{T}$-mixed Poisson structures (see $\oint 2.3)$, we figure out the $T$-leaf decomposition of $\left(G / U, \pi_{G / U}\right)$.

Let $X$ be a scheme defined over an algebraically closed field $k$ of positive characteristics. In 1985, Mehta and Ramanathan introduced in [19] the notion of Frobenius splittings on $X$ as a $\mathcal{O}_{X}$-linear map $\varphi: F_{*} \mathcal{O}_{X} \rightarrow \mathcal{O}_{X}$ that splits $F^{\sharp}: \mathcal{O}_{X} \rightarrow F_{*} \mathcal{O}_{X}$, where $F$ is the Frobenius endomorphism of $X$. They proved that both Bott-Samelson varieties and Schubert varieties are Frobenius split and obtained a cohomology vanishing result for line bundles on both BottSamelson varieties and Schubert varieties as a consequence. See [13, 19] for references. The

\footnotetext{
${ }^{0}$ Keywords: Frobenius splittings, Poisson $\mathbb{T}$-Pfaffians, the basic affine space

${ }^{0} M S C: 14 \mathrm{~F} 17,20 \mathrm{G} 05$, 53D17
} 
notion of Poisson $\mathbb{T}$-Pfaffian can be also defined on nonsingular $\mathbb{T}$-Poisson varieties defined over an algebraically closed field of positive characteristic (see Definition 1.2). Recall that a Frobenius near-splitting on $X$ is an $\mathcal{O}_{X}$-linear map $\varphi: F_{*} \mathcal{O}_{X} \rightarrow \mathcal{O}_{X}$. Given a Poisson $\mathbb{T}$-Pfaffian $\sigma$ on a $\mathbb{T}$-Poisson variety $\left(X, \pi_{X}\right)$, one can then use $\sigma^{p-1}$ to define a Frobenius near-splitting, where $p=$ char $k$. Furthermore, we prove that when the near-splitting is a splitting, then all compatibly split subvarieties of the splitting must be $\mathbb{T}$-Poisson subvarieties.

It is well-known that complex simple Lie algebras are classified into types $A, B, C, D, E, F, G$. Over an algebraically closed field $k$ of characteristic $p>0$, recall from [10, §25.4] that a Chevalley algebra can be constructed via reduction modulo $p$ by choosing a Chevalley basis of a complex semi-simple Lie algebra. Then, Chevalley groups are obtained from Chevalley algebras and any simply-connected semi-simple algebraic group $G$ over $k$ is isomorphic to one of the Chevalley groups. See [4] and [10, §25.5] for more details. Assume additionally that $p>3$, our general theory can be applied to show that Frobenius splittings can be constructed on Bott-Samelson varieties via Poisson geometry, which share the same property as the canonical splittings constructed in [2, Theorem 2.2.3] that all the sub-Bott-Samelson varieties are compatibly-split. Lastly, we apply our general theory to reach our goal, which is to construct a Frobenius splitting on $G / U$ via Poisson geometry.

1.2. Poisson geometry of $\left(G / U, \pi_{G / U}\right)$. When $k=\mathbb{C}$, let $\mathfrak{g}$ be the Lie algebra of $G$. Let $\left(B, B_{-}\right)$be a pair of opposite Borel subgroups of $G$. Let $T=B \cap B_{-}$a maximal torus of $G$ whose Lie algebra is denoted by $\mathfrak{h}$. Let $\langle,\rangle_{\mathfrak{g}}$ be a multiple of Killing form on $\mathfrak{g}$. Let $\pi_{\text {st }}$ be the standard Poisson structure on $G$ (see $\oint 2.2$ for detail), which is preserved under the left translation of $T$. The Poisson structure $\pi_{\text {st }}$ projects to a $T$-invariant Poisson structure on $G / U$, denoted as $\pi_{G / U}$. We call $\pi_{G / U}$ the standard Poisson structure on $G / U$.

Let $W=N_{G}(T) / T$ be the Weyl group of $(G, T)$, where $N_{G}(T)$ is the normalizer of $T$ in $G$. On $G / U$, there exists the Bruhat decomposition

$$
G / U=\bigsqcup_{w \in W} B w B / U
$$

such that each $B w B / U$ is a Poisson submanifold of $\left(G / U, \pi_{G / U}\right)$. On each $B w B / U$, there exists an isomorphism

$$
\kappa_{\dot{w}}: B w B / U \rightarrow T \times(B w B / B)
$$

defined in (2.7). On $T \times(B w B / B)$, there exists a $\mathbb{T}$-mixed Poisson structure defined by

$$
0 \bowtie_{A_{0}} \pi_{G / B}=\left(\left(0, \pi_{G / B}\right)+\sum_{k}\left(\rho\left(H_{k}\right), 0\right) \wedge\left(0, \lambda\left(H_{k}\right)\right),\right.
$$

where $\pi_{G / B}$ is the projection of $\pi_{\mathrm{st}}$ to $G / B,\left(H_{k}\right)$ is an orthogonal basis of $\left(\mathfrak{h},\langle,\rangle_{\mathfrak{g}}\right), \rho$ and $\lambda$ are natural Lie algebra actions of $\mathfrak{h}$ on $T$ and $B w B / B$ respectively.

The following Theorem A is the main tool of this paper in Poisson geometry which is applied in both Theorem B and Theorem E.

Theorem A. The isomorphism

$$
\kappa_{\dot{w}}:\left(B w B / U, \pi_{G / U}\right) \rightarrow\left(T \times(B w B / B), 0 \bowtie_{A_{0}} \pi_{G / B}\right)
$$


is Poisson.

Let $\left(X, \pi_{X}\right)$ be a $\mathbb{T}$-Poisson manifold. Suppose that $\Sigma$ is a symplectic leaf in $\left(X, \pi_{X}\right)$. The set

$$
\mathbb{T} \Sigma=\bigsqcup_{t \in \mathbb{T}} t \Sigma
$$

is called a single $\mathbb{T}$-leaf in $\left(X, \pi_{X}\right)$. See [16, Section 2.2] for more details.

The left translation of $T$ on $G / U$ makes $\left(G / U, \pi_{G / U}\right)$ into a natural $T$-Poisson manifold. Using Theorem A, we figure out the $T$-leaves decomposition of $\left(G / U, \pi_{G / U}\right)$.

Theorem B. The decomposition of $G / U$ into T-leaves of the Poisson structure $\pi_{G / U}$ is

$$
G / U=\bigsqcup_{v \leq u}\left(B u B / U \cap B_{-} v B / U\right) .
$$

1.3. General construction of Frobenius splittings via Poisson geometry. To study Frobenius splittings on $G / U$, where $G$ is defined over an algebraically closed field $k$ of characteristic $p>3$, we first develop a general construction via Poisson geometry. The details about Poisson algebras in positive characteristics are presented in $\$ 3.1$.

Definition 1.1. Let $X$ be a non-singular variety over $k$. A Poisson structure on $X$ is a section $\pi \in H^{0}\left(X, \wedge^{2} \mathcal{T}_{X}\right)$ satisfying $[\pi, \pi]=0$, where $[-,-]$ is the Schouten bracket (see $\$ 3.1$ for the definition of the Schouten bracket). A (non-singular) Poisson variety is a non-singular variety equipped with a Poisson structure.

Definition 1.2. Let $\mathbb{T}$ be an algebraic torus over $k$. A $\mathbb{T}$-Poisson variety is a Poisson variety $X$ with a $\mathbb{T}$-action by Poisson automorphisms. A Poisson $\mathbb{T}$-Pfaffian is a section $\sigma \in H^{0}\left(X, \omega_{X}^{-1}\right)$ such that

$$
\sigma=\pi^{[r]} \wedge \partial_{h_{1}} \wedge \ldots \wedge \partial_{h_{l}}
$$

where $r$ is half of the rank of $\pi$ and $\partial_{h_{i}}$ is the vector field on $X$ generated by some element $h_{i} \in \mathfrak{t}=\operatorname{Lie}(\mathbb{T})$. (See Definition 3.10 for the definition of generating vector fields)

Let $s \in H^{0}\left(X, \omega_{X}^{1-p}\right)$. One can regard $s$ as an element in $\operatorname{Hom}_{\mathcal{O}_{\mathrm{X}}}\left(\omega_{\mathrm{X}}, \mathrm{F}_{*} \omega_{\mathrm{X}}\right)$ by $s(\gamma)=$ $\left(\left.s\right|_{U}, \gamma^{p-1}\right) \gamma$, for $\gamma \in H^{0}\left(\mathcal{U}, \omega_{X}\right)$, where $\mathcal{U}$ is an open subset of $X$. It is well-known that the Cartier operator gives rise to the isomorphism ([2, Proposition 1.3.7])

$$
\mathcal{H o m}_{\mathcal{O}_{X}}\left(\omega_{X}, F_{*} \omega_{X}\right) \cong \mathcal{H o m}_{\mathcal{O}_{X}}\left(F_{*} \mathcal{O}_{X}, \mathcal{O}_{X}\right) \text {. }
$$

Therefore in particular the $p-1$ tensor power $\sigma^{p-1}$ of a Poisson $\mathbb{T}$-Pfaffian $\sigma$ gives rise to a Frobenius near-splitting $\phi_{\sigma}$ of $X$.

Definition 1.3. A Frobenius Poisson $\mathbb{T}$-Pfaffian of a $\mathbb{T}$-Poisson variety $\left(X, \pi_{X}\right)$ is a Poisson $\mathbb{T}$ Pfaffian $\sigma$ such that the Frobenius near-splitting constructed in the way above by some non-zero scalar multiple of $\sigma$ is a Frobenius splitting.

Remark. A basic fact about Frobenius splitting is that if one can verify the restriction of a near-splitting $\varphi$ on a variety $X$ to an open subset $\mathcal{U}$ of $X$ is a Frobenius splitting on $\mathcal{U}$, then $\varphi$ is a Frobenius splitting on $X$. See [2, Section 1.1] for more details. The advantage of constructing the near-splitting via Poisson $\mathbb{T}$-Pfaffian is that often there exists some nice expression of the Poisson structure in local coordinates. 
In $₫ 3.3$ we consider a special kind of $\mathbb{T}$-Poisson structure on affine spaces called Poisson CGL extensions, a term coined by K.Goodearl and M.Yakimov and named after G.Cauchon, K.Goodearl, E.Letzter ([9, Section 1.3]). They play an important role in the study of cluster algebras (see [9]) as well as integrable systems (see [14]). We define the notion of a Poisson CGL bivector field in Definition 3.19 and its log-canonical part in Remark 3.20, Then, we obtain a sufficient condition such that a Frobenius Poisson $\mathbb{T}$-Pfaffian exists on a $\mathbb{T}$-Poisson variety.

Theorem C. If the rank of a Poisson CGL bivector field $\pi_{X}$ is equal to that of $\left(\pi_{X}\right)_{0}$, the log-canonical part of $\pi_{X}$, then a Frobenius Poisson $\mathbb{T}$-Pfaffian exists.

For a general $\mathbb{T}$-Poisson variety $\left(X, \pi_{X}\right)$, the connection between compatibly split subvarieties of $X$ and $\mathbb{T}$-Poisson subvarieties of $\left(X, \pi_{X}\right)$ is presented in the following Theorem $\mathrm{D}$.

Theorem D. Let $\sigma$ be a Poisson $\mathbb{T}$-Pfaffian of a $\mathbb{T}$-Poisson variety $\left(X, \pi_{X}\right)$. If the Frobenius near-splitting $\phi_{\sigma}$ defined by $\sigma$ is a Frobenius splitting, then all compatibly split subvarieties of $\phi_{\sigma}$ must also be $\mathbb{T}$-Poisson subvarieties with respect to the Poisson structure $\pi_{X}$.

1.4. A Frobenius Splitting of $G / U$ via Poisson $T$-Pfaffian. Theorem $\mathrm{C}$ can be applied to concrete examples including Bott-Samelson varieties (see 4.1 for the definition of Bott-Samelson varieties) and the basic affine space when $k$ is of characteristic $p>3$.

First, we equip the Bott-Samelson varieties with the standard Poisson structure as well as a torus action (see the paragraph below Remark 4.2 for the definition of the standard Poisson structure of Bott-Samelson varieties and basic affine spaces over positive characteristic). Then, Theorem 4.3 proves that a Frobenius Poisson $T$-Pfaffian exists on any Bott-Samelson varieties and is unique up to scalar multiple. Therefore, a Frobenius splitting $\phi_{\sigma}$ defined in $\$ 1.3$ exists on any Bott-Samelson variety. Moreover, Frobenius splittings on Bott-Samelson varieties can be also obtained from a class of special anti-canonical sections by [2, Theorem 2.2.3] and the canonical Frobenius splittings are constructed in the proof of [2, Theorem 2.2.3]. On any BottSamelson variety, the Frobenius Poisson $T$-Pfaffian constructed in Theorem 4.3 provides a choice of anti-canonical section in [2, Theorem 2.2.3] and a natural question is raised as follows.

Question 1.4. On any Bott-Samelson variety, is the Frobenius splitting induced by the Poisson T-Pfaffian in Theorem 4.3 same as the Frobenius splitting constructed in the proof of [2, Theorem 2.2.3] ?

Based on Theorem 4.3 and Theorem A, we reach our goal of this paper, which is to construct a Frobenius splitting on $G / U$ via the Poisson geometry as follows.

Theorem E. Let $G$ be a simply-connected semi-simple algebraic group over an algebraically closed field $k$ of characteristic $p>3, U$ the uniradical of a Borel subgroup of $G$. Let $T$ be a maximal torus of $G$ normalizing $U$, which acts on $G / U$ by left translation. Denote by $\pi_{G / U}$ the standard Poisson structure on $G / U$. Then there exists a unique (up to scalar multiples)

Poisson T-Pfaffian $\sigma$ on $\left(G / U, \pi_{G / U}\right)$ and $\sigma^{p-1}$ induces a Frobenius splitting on $G / U$.

1.5. Acknowledgments. The authors would like to thank Jiang-Hua Lu for her help and encouragements. This work was completed while the authors were supported by the University of Hong Kong Postgraduate Studentship. 


\section{Poisson geometry of $\left(G / U, \pi_{G / U}\right)$}

2.1. Basics on Poisson geometry. Let $G$ be a complex Lie group and let $\pi_{G}$ be a holomorphic Poisson structure on $G$. The pair $\left(G, \pi_{G}\right)$ is called a Poisson Lie group if the multiplication map

$$
\left(G \times G, \pi_{G} \times \pi_{G}\right) \rightarrow\left(G, \pi_{G}\right)
$$

is Poisson. See [8, 17] for references. It is well known (see [17, Theorem 1.2]) that $\left(G, \pi_{G}\right)$ is a Poisson Lie group if and only if $\pi_{G}$ is multiplicative, i.e.

$$
\pi_{G}(g h)=l_{g} \pi_{G}(h)+r_{h} \pi_{G}(g), \quad g, h \in G .
$$

Lemma 2.1. [17, Theorem 1.2] Let $\left(G, \pi_{G}\right)$ be a Poisson Lie group. Then, $\pi_{G}$ vanishes at the identity and the group inversion map is anti-Poisson.

Let $\left(G, \pi_{G}\right)$ be a complex Poisson Lie group and let $\left(P, \pi_{P}\right)$ be a complex Poisson variety such that $G$ acts on $P$ from the left preserving the Poisson structure. If the action is transitive, $\left(P, \pi_{P}\right)$ is called a Poisson homogeneous space of $\left(G, \pi_{G}\right)$. See [6] for reference.

A subgroup/variety $Q$ of a Poisson Lie group/variety $\left(G, \pi_{G}\right)$ is called a coisotropic subgroup/variety if for any $q \in Q$

$$
\pi_{G}(q) \in T_{q} Q \wedge T_{q} G \subset \wedge^{2} T_{q} G .
$$

The following proposition proved in [21, Theorem 6] provides a class of examples of Poisson homogeneous spaces.

Proposition 2.2. Let $\left(G, \pi_{G}\right)$ be a Poisson Lie group and let $Q \subset G$ be a closed coisotropic Lie subgroup of $\left(G, \pi_{G}\right)$. Denote the projection from $G$ to $G / Q$ by $p_{G / Q}$. Then $p_{G / Q}\left(\pi_{G}\right)$ is a well defined Poisson structure on $G / Q$ and $\left(G / Q, p_{G / Q}\left(\pi_{G}\right)\right)$ is a Poisson homogeneous space of $\left(G, \pi_{G}\right)$.

Let $\mathbb{T}$ be a complex torus. Recall that a complex $\mathbb{T}$-Poisson variety is a Poisson variety $\left(P, \pi_{P}\right)$ with a $\mathbb{T}$-action by Poisson automorphisms. Let $\mathbb{T}_{1}$ and $\mathbb{T}_{2}$ be complex tori with Lie algebras $\mathfrak{t}_{1}$ and $\mathfrak{t}_{2}$ respectively. Let $\left(P, \pi_{P}\right)$ be a complex $\mathbb{T}_{1}$-Poisson variety and $\left(Q, \pi_{Q}\right)$ be a complex $\mathbb{T}_{2}$-Poisson variety. The $\mathbb{T}_{1}$ and $\mathbb{T}_{2}$ actions are denoted by $\rho$ and $\lambda$ respectively. Given

$$
A=\sum_{k} a_{k} \otimes b_{k} \in \mathfrak{t}_{1} \otimes \mathfrak{t}_{2},
$$

define the bivector field on $P \times Q$ by

$$
\pi_{P} \bowtie_{A} \pi_{Q}=\left(\pi_{P}, 0\right)+\left(0, \pi_{Q}\right)+\sum_{k}\left(\rho\left(a_{k}\right), 0\right) \wedge\left(0, \lambda\left(b_{k}\right)\right) .
$$

Consider the $\left(\mathbb{T}_{1} \times \mathbb{T}_{2}\right)$ action on $P \times Q$ defined by

$$
\left(t_{1}, t_{2}\right)(p, q)=\left(t_{1} p, t_{2} q\right)
$$

The following lemma proved in [24, Lemma 2.1.1].

Lemma 2.3. For any $A \in \mathfrak{t}_{1} \otimes \mathfrak{t}_{2},\left(P \times Q, \pi_{P} \bowtie_{A} \pi_{Q}\right)$ is a $\left(\mathbb{T}_{1} \times \mathbb{T}_{2}\right)$-Poisson variety.

The mixed product Poisson structure on $P \times Q$ associated to the torus actions constructed in Lemma 2.3 on $P \times Q$ is simply called a $\mathbb{T}$-mixed Poisson structure. 
2.2. The standard Poisson structure $\pi_{G / U}$ on $G / U$. Let $G$ be a simply-connected complex semi-simple Lie group and let $\mathfrak{g}$ be the Lie algebra of $G$. Let $\left(B, B_{-}\right)$be a pair of opposite Borel subgroups of $G$. Denote $U$ and $U_{-}$respectively the uniradical of $B$ and $B_{-}$. Let $T=B \cap B_{-}$ be a maximal torus of $G$. Denote Lie algebras of $B, B_{-}, U, U_{-}$and $T$ by $\mathfrak{b}, \mathfrak{b}_{-}, \mathfrak{u}, \mathfrak{u}_{-}$and $\mathfrak{h}$ respectively. Let $\Delta \subset \mathfrak{h}^{*}$ be the set of roots of $\mathfrak{g}$ with respect to $\mathfrak{h}$, and let

$$
\mathfrak{g}=\mathfrak{h}+\sum_{\alpha \in \Delta} \mathfrak{g}_{\alpha}
$$

be the root decomposition of $\mathfrak{g}$. Let $\Delta_{+} \subset \Delta$ and $\Gamma \subset \Delta_{+}$be respectively the sets of positive roots and simple roots. We also write $\alpha>0$ for $\alpha \in \Delta_{+}$.

For $\alpha>0$, let $h_{\alpha}$ be the unique element in $\left[\mathfrak{g}_{\alpha}, \mathfrak{g}_{-\alpha}\right]$ such that $\alpha\left(h_{\alpha}\right)=2$. Fix, for each $\alpha \in \Delta_{+}, e_{\alpha} \in \mathfrak{g}_{\alpha}$ and $e_{-\alpha} \in \mathfrak{g}_{-\alpha}$ such that $\left[e_{\alpha}, e_{-\alpha}\right]=h_{\alpha}$. Let $\langle,\rangle_{\mathfrak{g}}$ be a fixed multiple of Killing form on $\mathfrak{g}$ such that $\frac{\langle\alpha, \alpha\rangle}{2} \in\{1,2,3\}$ for each root $\alpha$, where $\langle$,$\rangle is the induced bilinear$ form on $\mathfrak{h}^{*}$.

Remark 2.4. The existence of such choice follows from the proof of [22, Lemma 5].

For $\alpha>0$, denote $E_{\alpha}=\sqrt{\langle\alpha, \alpha\rangle} e_{\alpha}$ and $E_{-\alpha}=\frac{\sqrt{\langle\alpha, \alpha\rangle}}{2} e_{-\alpha}$. Let $\left(H_{i}\right)$ be an orthogonal basis of $\left(\mathfrak{h},\langle,\rangle_{\mathfrak{g}}\right)$.

Definition 2.5. [8, Chapter 4][16, Section 6.1] The standard Poisson structure $\pi_{\mathrm{st}}$ on $G$ is defined by

$$
\pi_{\mathrm{st}}(g)=l_{g} \Lambda_{\mathrm{st}}-r_{g} \Lambda_{\mathrm{st}}, g \in G
$$

where $l_{g}$ and $r_{g}$ are respectively the left and right translation of $g$ on $G$ and

$$
\Lambda_{\mathrm{st}}=\sum_{\alpha>0} \frac{\langle\alpha, \alpha\rangle}{2} e_{-\alpha} \wedge e_{\alpha}=\sum_{\alpha>0} E_{-\alpha} \wedge E_{\alpha}
$$

Remark 2.6. The Poisson structure $\pi_{\text {st }}$ depends on the choices of the triple $\left(B, B_{-},\langle,\rangle_{\mathfrak{g}}\right)$, but not on the choices of the root vectors $e_{ \pm \alpha} \in \mathfrak{g}_{ \pm \alpha}$.

Recall that in characteristic $p>3$, the Lie algebra of a simply-connected semi-simple algebraic group can be constructed by reduction modulo $p$ via a Chevalley basis of a complex semi-simple Lie algebra. Since we have chosen the bilinear form so that $\frac{\langle\alpha, \alpha\rangle}{2} \in\{1,2,3\}$ for each root $\alpha$ in the complex case, $\frac{\langle\alpha, \alpha\rangle}{2}$ is non-zero modulo $p$ and the element

$$
\Lambda_{\mathrm{st}}=\sum_{\alpha>0} \frac{\langle\alpha, \alpha\rangle}{2} e_{-\alpha} \wedge e_{\alpha}
$$

modulo $p$ is well-defined.

Definition 2.7. The standard Poisson structure on a simply-connected semi-simple algebraic group over an algebraically closed field of characteristic $p>3$ is defined by the same formula as in Definition 2.5.

For a root $\beta$, denote by $u_{\beta}(z)$ the root-subgroup corresponding to $\beta$, where $z \in \mathbb{C}$. For each $\alpha \in \Gamma$, the simple reflection $s_{\alpha} \in W$ is represented by

$$
\dot{s}_{\alpha}=u_{\alpha}(-1) u_{-\alpha}(1) u_{\alpha}(-1)
$$


in $N_{G}(T)$. We denote by $P_{\alpha}=B \cup B \dot{s}_{\alpha} B$ the parabolic subgroup corresponding to $\alpha \in \Gamma$. It is well-known that $P_{\alpha}$ is a Poisson-Lie subgroup of $G$ for all $\alpha$. We denote the restriction of $\pi_{\mathrm{st}}$ on $P_{\alpha}$ by the same notation. Let $\left(H_{i}\right)$ be an orthogonal basis of $\left(\mathfrak{h},\langle,\rangle_{\mathfrak{g}}\right)$. Consider now the direct product Lie algebra $\mathfrak{g} \oplus \mathfrak{g}$ and define $R_{\text {st }} \in(\mathfrak{g} \oplus \mathfrak{g})^{\otimes 2}$ by

$$
\begin{aligned}
R_{\mathrm{st}}=\sum_{\alpha \in \Delta_{+}}\left(E_{-\alpha}, E_{-\alpha}\right) \wedge\left(E_{\alpha}, 0\right)+\sum_{\alpha \in \Delta_{+}}\left(E_{\alpha}, E_{\alpha}\right) \wedge\left(0, E_{-\alpha}\right) & \\
& +\frac{1}{2} \sum_{i}\left(H_{i}, H_{i}\right) \wedge\left(H_{i},-H_{i}\right)
\end{aligned}
$$

and

$$
\Pi_{\mathrm{st}}=R_{\mathrm{st}}^{l}-R_{\mathrm{st}}^{r} .
$$

Then, $\left(G \times G, \Pi_{\text {st }}\right)$ is a Poisson Lie group, which is called the Drinfeld double of $\left(G, \pi_{\mathrm{st}}\right)$. See [18] for more details. Recall from [18, Proposition 2.2] that

$$
G_{\Delta}=\{(g, g): g \in G\} \subset G \times G
$$

is a Poisson Lie subgroup of $\left(G \times G, \Pi_{\text {st }}\right)$ satisfying the following Lemma 2.8 .

Lemma 2.8. The diagonal embedding

$$
\left(G, \pi_{\mathrm{st}}\right) \longrightarrow\left(G \times G, \Pi_{\mathrm{st}}\right), \quad g \longmapsto(g, g)
$$

is Poisson.

It follows from the construction of $\pi_{\text {st }}$ that $U$ is a coisotropic subgroup of $\left(G, \pi_{\text {st }}\right)$. Moreover, the following lemma is proved in [16, Section 6.1].

Lemma 2.9. The standard Poisson structure $\pi_{\mathrm{st}}$ vanishes on $T$ and $B w B$ is a Poisson subvariety of $\left(G, \pi_{\mathrm{st}}\right)$ for any $w \in W$.

Definition 2.10. The projection of $\pi_{\text {st }}$ to $G / U$ is a well-defined Poisson structure by Proposition 2.2, which is denoted by $\pi_{G / U}$ and called the standard Poisson structure on $G / U$.

Let $\lambda$ be the $T$-action on $G / U$ defined by

$$
T \times(G / U) \longrightarrow(G / U), \quad(t, g . U) \longmapsto t g . U, \quad t \in T, g \in G .
$$

Lemma 2.11. $\left(G / U, \pi_{G / U}\right)$ is a T-Poisson variety.

Proof. It follows from Proposition 2.2 that $\left(G / U, \pi_{G / U}\right)$ is a Poisson homogeneous space, i.e. for $t \in T$ and $g \in G$,

$$
\pi_{G / U}(\operatorname{tg} \cdot U)=\sigma_{g . U} \pi_{\mathrm{st}}(t)+\lambda_{t} \pi_{G / U}(g \cdot U)
$$

where $\sigma_{g . U}: T \rightarrow G / U, g . U \mapsto t^{\prime} g . U, \forall t^{\prime} \in T$, and $\lambda_{t}: G / U \rightarrow G / U, g^{\prime} . U \mapsto t g^{\prime} . U, \forall g^{\prime} \in G$. As $\pi_{\text {st }}$ vanishes on $T$, the left translation of $T$ on $G / U$ preserves the Poisson structure $\pi_{G / U}$, i.e.

$$
\pi_{G / U}(\operatorname{tg} \cdot U)=\lambda_{t} \pi_{G / U}(g \cdot U) .
$$

Q.E.D. 
2.3. Proof of Theorem A. Let $G$ be a complex semi-simple algebraic group and follow the same notation as in $\$ 2.2$. It is well-known that $G$ has the Bruhat decomposition

$$
G=\bigsqcup_{w \in W} B w B
$$

As $U$ is a closed subgroup of $B, G / U$ also has the Bruhat decomposition

$$
G / U=\bigsqcup_{w \in W} B w B / U
$$

For each $w \in W$, it follows from Lemma 2.9 that $B w B$ is a Poisson subvariety of $\left(G, \pi_{\mathrm{st}}\right)$ and $B w B / U$ is a Poisson subvariety of $\left(G / U, \pi_{G / U}\right)$. For $w \in W$, define $U_{w}=U \cap w U_{-} w^{-1}$. If we fix a representative $\dot{w} \in N_{G}(T)$, any $g \in B w B$ can be uniquely decomposed as $g=t x \dot{w} n$ with $t \in T, x \in U_{w}$ and $n \in U$. For any $w \in W$, consider the isomorphism on $B w B$ defined by

$$
J_{\dot{w}}: B w B \rightarrow T \times(B w B / T), \quad t x \dot{w} n \mapsto(t, x \dot{w} n \cdot T) .
$$

and the isomorphism on $B w B / U$ defined by

$$
\kappa_{\dot{w}}: B w B / U \rightarrow T \times(B w B / B), \quad t x \dot{w} \cdot U \mapsto(t, x w \cdot B) .
$$

Recall from Proposition 2.2 that $\pi_{\text {st }}$ also projects to well-defined Poisson structures on $G / T$ as well as $G / B$, denoted respectively by $\pi_{G / T}$ and $\pi_{G / B}$. Let $\rho$ be the torus action defined by

$$
T \times T \longrightarrow T, \quad\left(t, t^{\prime}\right) \longmapsto t t^{\prime} .
$$

When $Q=B$ or $T$, let $\lambda$ be the torus action defined by

$$
T \times(B w B / Q) \longrightarrow(B w B / Q), \quad(t, g \cdot Q) \longmapsto t g \cdot Q .
$$

Let $\left(H_{i}\right)$ be an orthogonal basis of $\left(\mathfrak{h},\langle,\rangle_{\mathfrak{g}}\right)$ and let

$$
A_{0}=\sum_{i} H_{i} \otimes H_{i}
$$

Similar to the proof of Lemma 2.11, we know that $\left(G / Q, \pi_{G / Q}\right)$ is a $T$-Poisson variety. Then, $0 \bowtie_{A_{0}} \pi_{G / Q}$ defined in (2.2) is a $\mathbb{T}$-mixed Poisson structure on $T \times(B w B / Q)$.

To prove Theorem A, we first prove the following Proposition 2.12, which expresses $J_{\dot{w}}\left(\pi_{\mathrm{st}}\right)$ as a $\mathbb{T}$-mixed Poisson structure on $T \times(B w B / T)$.

Proposition 2.12. The isomorphism

$$
J_{\dot{w}}:\left(B w B, \pi_{\mathrm{st}}\right) \rightarrow\left(T \times(B w B / T), 0 \bowtie_{A_{0}} \pi_{G / T}\right)
$$

is Poisson.

Proof. First, we prove that the map

$$
q_{\dot{w}}:\left(B w B, \pi_{\mathrm{st}}\right) \rightarrow(T, 0), \quad t x \dot{w} n \mapsto t
$$

is Poisson. For any $g \in B w B$ and a fixed $\dot{w} \in N_{G}(T), g$ can be uniquely written as

$$
g=t x \dot{w} n=t n^{\prime} \dot{w} x^{\prime}
$$

for the same $t \in T, x \in U_{w}, x^{\prime} \in U \cap w^{-1} U_{-} w$ and $n, n^{\prime} \in U$. So, we can write

$$
q_{\dot{w}}=j_{T} \circ j_{\dot{w}},
$$


where

$$
j_{\dot{w}}:\left(B w B, \pi_{\mathrm{st}}\right) \rightarrow\left(B, \pi_{\mathrm{st}}\right), t n^{\prime} \dot{w} x^{\prime} \mapsto t n^{\prime}, t \in T, n^{\prime} \in U, x^{\prime} \in U \cap w^{-1} U_{-} w,
$$

and

$$
j_{T}:\left(B, \pi_{\mathrm{st}}\right) \rightarrow(T, 0), t n^{\prime} \mapsto t, t \in T, n^{\prime} \in U .
$$

It is sufficient to prove that $j_{\dot{w}}$ and $j_{T}$ are Poisson.

By the multiplicativity of $\pi_{\text {st }}$, one has

$$
\pi_{\mathrm{st}}(t x \dot{w} n)=\pi_{\mathrm{st}}\left(t n^{\prime} \dot{w} x^{\prime}\right)=l_{t n^{\prime}} \pi_{\mathrm{st}}\left(\dot{w} x^{\prime}\right)+r_{\dot{w} x^{\prime}} \pi_{\mathrm{st}}\left(t n^{\prime}\right),
$$

where $r_{g}$ denotes the right translation on $G$ and $l_{h}$ denotes the left translation on $G$. On one hand, for a fixed $t n^{\prime} \in B$, all the elements in $\dot{w} U \cap U_{-} \dot{w}$ go to the same point $t n^{\prime}$ via $j_{\dot{w}} \circ l_{t n^{\prime}}$, i.e. for any $\dot{w} x^{\prime} \in \dot{w} U \cap U_{-} \dot{w}$

$$
j_{\dot{w}} l_{t n^{\prime}}\left(\dot{w} x^{\prime}\right)=t n^{\prime} .
$$

As $\dot{w} U \cap U_{-} \dot{w}$ is coisotropic in $\left(G, \pi_{\text {st }}\right)$, (see [15, Lemma 10]) one has

$$
j_{\dot{w}} l_{t n^{\prime}} \pi_{\mathrm{st}}\left(\dot{w} x^{\prime}\right)=0 .
$$

On the other hand, for a fixed $\dot{w} \in N_{G}(T)$ and $x^{\prime} \in U \cap w^{-1} U_{-} w, j_{\dot{w}} \circ r_{\dot{w} x^{\prime}}$ fixes $B$ pointwise, i.e. for any $t n^{\prime} \in B$,

$$
j_{\dot{w}} \circ r_{\dot{w} x^{\prime}}\left(t n^{\prime}\right)=t n^{\prime} .
$$

As $B$ is a Poisson Lie subgroup of $\left(G, \pi_{\mathrm{st}}\right)$, one has

$$
j_{\dot{w}}\left(\pi_{\mathrm{st}}\left(t n^{\prime} \dot{w} x^{\prime}\right)\right)=j_{\dot{w}} r_{\dot{w} x^{\prime}} \pi_{\mathrm{st}}\left(t n^{\prime}\right)=\pi_{\mathrm{st}}\left(t n^{\prime}\right) .
$$

Moreover, as $U$ is a coisotropic subgroup of $\left(B, \pi_{\mathrm{st}}\right)$ and $\pi_{\mathrm{st}}$ vanishes on $T$, one has

$$
j_{T} \pi_{\mathrm{st}}\left(t n^{\prime}\right)=j_{T} l_{t} \pi_{\mathrm{st}}\left(n^{\prime}\right)=0 .
$$

Therefore, $j_{\dot{w}}$ and $j_{T}$ are Poisson maps.

Then, we study the mixed terms. Define

$$
\Psi_{\dot{w}}:(B w B) \times G \rightarrow T \times(G / T), \quad(t x \dot{w} n, g) \mapsto(t, g \cdot T) .
$$

Recall from Lemma 2.8 that $\left(B w B, \pi_{\mathrm{st}}\right)$ is Poisson isomorphic to $\left((B w B)_{\Delta}, \Pi_{\mathrm{st}}\right)$, where

$$
(B w B)_{\Delta}=\{(g, g): g \in B w B\} \subset G_{\Delta} .
$$

We can identify $J_{\dot{w}} \pi_{\text {st }}$ with $\Psi_{\dot{w}}\left(\Pi_{\text {st }}\right)$. By the definition of $\Pi_{\text {st }}$, write

$$
\Pi_{\mathrm{st}}=\left(\pi_{\mathrm{st}}, 0\right)+\left(0, \pi_{\mathrm{st}}\right)+\mu_{1}+\mu_{2}+\mu_{3}+\mu_{4}
$$

where,

$$
\mu_{1}=2 \sum_{\alpha \in \Delta_{+}}\left(E_{\alpha}^{r}, 0\right) \wedge\left(0, E_{-\alpha}^{r}\right), \quad \mu_{2}=-2 \sum_{\alpha \in \Delta_{+}}\left(E_{\alpha}^{l}, 0\right) \wedge\left(0, E_{-\alpha}^{l}\right)
$$

and

$$
\mu_{3}=\sum_{i=1}^{n}\left(H_{i}^{r}, 0\right) \wedge\left(0, H_{i}^{r}\right), \quad \mu_{4}=-\sum_{i=1}^{n}\left(H_{i}^{l}, 0\right) \wedge\left(0, H_{i}^{l}\right) .
$$


Since the vector fields $E_{\alpha}^{l}, E_{\alpha}^{r}$ vanish when projected to $T$ and $H_{i}^{l}$ on $B w B$ vanishes when projected to $B w B / T$, one has $\Psi_{\dot{w}}\left(\mu_{1}\right)=\Psi_{\dot{w}}\left(\mu_{2}\right)=\Psi_{\dot{w}}\left(\mu_{4}\right)=0$. Additionally, one has

$$
\Psi_{\dot{w}}\left(\mu_{3}\right)=\sum_{i}\left(\rho\left(H_{i}\right), 0\right) \wedge\left(0, \lambda\left(H_{i}\right)\right)
$$

where $\rho$ and $\lambda$ are Lie algebra actions induced by (2.8) and (2.9) respectively. Therefore, $J_{\dot{w}} \pi_{\text {st }}=$ $0 \bowtie A_{0} \pi_{G / T}$.

\section{Q.E.D.}

Furthermore, the following theorem expresses $\kappa_{\dot{w}}\left(\pi_{G / U}\right)$ as a $\mathbb{T}$-mixed Poisson structure on $T \times(B w B / B)$.

Theorem 2.13. The isomorphism

$$
\kappa_{\dot{w}}:\left(B w B / U, \pi_{G / U}\right) \rightarrow\left(T \times(B w B / B), 0 \bowtie_{A_{0}} \pi_{G / B}\right)
$$

is Poisson.

Proof. Let $p_{G / U}$ be the natural projection from $G$ to $G / U$. When restricted to $B w B$, one has

$$
J_{\dot{w}} \circ p_{G / U}=p_{G / U} \circ J_{\dot{w}} .
$$

Therefore, on each $B w B / U$, one has

$$
\kappa_{\dot{w}}\left(\pi_{G / U}\right)=J_{\dot{w}} \circ p_{G / U}\left(\pi_{\mathrm{st}}\right)=p_{G / U}\left(J_{\dot{w}}\left(\pi_{\mathrm{st}}\right)\right)=0 \bowtie_{A_{0}} \pi_{G / U} .
$$

\section{Q.E.D.}

2.4. Proof of Theorem B. In [16, Section 1.5], J.-H. Lu and V. Mouquin develop a general theory to study the $\mathbb{T}$-leaves of a class of $\mathbb{T}$-Poisson manifolds. Let $G$ be a simply-connected complex semi-simple group. The general theory is applied to study the $T$-leaves of $\left(G / B, \pi_{G / B}\right)$ in [16, Theorem 1.1].

Proposition 2.14. [16, Theorem 1.1] The T-leaf decomposition of $\left(G / B, \pi_{G / B}\right)$ coincides with the disjoint union of open Richardson varieties

$$
G / B=\bigsqcup_{v \leq u}\left(B u B / B \cap B_{-} v B / B\right)
$$

However, the general theory cannot be applied to study the $T$-leaves of $\left(G / U, \pi_{G / U}\right)$. Based on the work in $\$ 2.3$, we give a proof of Theorem B. First, we recall the following Proposition 2.15 proved by J.-H. Lu and Y. Mi in [14, Lemma 2.23].

Proposition 2.15. Let $\mathbb{T}$ be a complex torus and $\left(X, \pi_{X}\right)$ a complex $\mathbb{T}$-Poisson manifold. Let $\mathfrak{t}$ be the Lie algebra of $\mathbb{T}$ and $A \in \mathfrak{t} \otimes \mathfrak{t}$. With respect to the diagonal action of $\mathbb{T}$ on $\mathbb{T} \times X$, the $\mathbb{T}$-leaves of the Poisson structure $0 \bowtie_{A} X$ in $\mathbb{T} \times X$ are precisely all the $\mathbb{T} \times L$, where $L$ is a $\mathbb{T}$-leaf of $\pi_{X}$ in $X$.

Theorem 2.16. The decomposition of $G / U$ into T-leaves of the Poisson structure $\pi_{G / U}$ is

$$
G / U=\bigsqcup_{v \leq u}\left(B u B / U \cap B_{-} v B / U\right)
$$


Proof. Recall that

$$
G / U=\bigsqcup_{u \in W} B u B / U
$$

such that $B u B$ is a Poisson subvariety of $\left(G / U, \pi_{G / U}\right)$ for each $u \in W$. To study the $T$-leaf decomposition of $\left(G / U, \pi_{G / U}\right)$, it is sufficient to study the $T$-leaves of $\left(B u B / U, \pi_{G / U}\right)$ for each fixed $u \in W$. It follows from Proposition 2.13, Proposition 2.14 and Proposition 2.15 that for each $v \leq u \in W$, the subvariety

$$
\kappa_{\dot{w}}^{-1}\left(T \times\left(B u B / B \cap B_{-} v B / B\right)\right)=B u B / U \cap B_{-} v B / U
$$

is a single $T$-leaf of $\left(B u B / U, \pi_{G / U}\right)$. Therefore, (2.12) gives $T$-leaf decomposition of $\left(G / U, \pi_{G / U}\right)$.

\section{Q.E.D.}

\section{General construction of Frobenius splittings via Poisson T-Pfaffians}

3.1. Poisson algebras in positive characteristics. Denote by $k$ an algebraically closed field with $\operatorname{char}(k)=p>2$. The purpose of this section is to explain a few aspects of Poisson algebras over $k$, and in particular the notion of Poisson $\mathbb{T}$-Pfaffians, $\mathbb{T}$ being an algebraic torus, which in the case of characteristic 0 is introduced in [16]. Note that a special feature is that, given a Poisson algebra $A$ and any element $a \in A$, the ideal $\left\langle a^{p}\right\rangle$ is a Poisson ideal since

$$
\left\{a^{p} b, c\right\}=a^{p}\{b, c\}+p a^{p-1} b\{a, c\}=a^{p}\{b, c\} \in\left\langle a^{p}\right\rangle, \quad b, c \in A
$$

Notation 3.1. Let $A$ be a $k$-algebra and $n$ be a non-negative integer. Recall that an $n$-derivation on $A$ is an element $\alpha \in \operatorname{Hom}_{\mathrm{k}}\left(\wedge^{\mathrm{n}} \mathrm{A}, \mathrm{A}\right)$ such that $\alpha$ is a derivation in each of its arguments. Following the notation in [12, Section 3.1], the vector space consisting of all $n$-derivations on $A$ will be denoted by $\mathfrak{X}^{n}(A)$. By convention, $\mathfrak{X}^{0}(A)=A$.

There is a natural $A$-module structure on $\mathfrak{X}^{n}(A)$ : for $b \in A$ and $\alpha \in \mathfrak{X}^{n}(A)$,

$$
(b \alpha)\left(a_{1}, \ldots, a_{n}\right)=b\left(\alpha\left(a_{1}, \ldots, a_{n}\right)\right), a_{1}, \ldots, a_{n} \in A
$$

Suppose $A$ is finitely generated by $r$ elements. By the skew-symmetry of multi-derivations, $\mathfrak{X}^{n}(A)=0$ for all $n>r$. Hence there exists a largest $n$ such that $\mathfrak{X}^{n}(A) \neq 0$. The module of top degree multi-derivations on a finitely generated $k$-algebra $A$, denoted by $\mathfrak{X}^{t o p}(A)$, is then defined to be $\mathfrak{X}^{n}(A)$ for such largest $n$.

We now briefly recall the definition of the wedge product and the Schouten bracket on $\mathfrak{X}(A)=$ $\bigoplus_{n=0}^{\infty} \mathfrak{X}^{n}(A)$. Let $\alpha \in \mathfrak{X}^{m}(A), \beta \in \mathfrak{X}^{n}(A)$ and $a_{1}, \ldots, a_{m+n} \in A$. Then $\alpha \wedge \beta \in \mathfrak{X}^{m+n}(A)$ is defined by

$$
(\alpha \wedge \beta)\left(a_{1}, \ldots, a_{m+n}\right)=\sum_{\sigma \in S_{m, n}} \operatorname{sgn}(\sigma) \alpha\left(a_{\sigma(1)}, \ldots, a_{\sigma(m)}\right) \beta\left(a_{\sigma(m+1)}, \ldots, a_{\sigma(m+n)}\right)
$$


where $S_{m, n}$ denotes the set of all $(m, n)$-shuffles, while $[\alpha, \beta] \in \mathfrak{X}^{m+n-1}(A)$ is defined by

$$
\begin{aligned}
& {[\alpha, \beta]\left(a_{1}, \ldots, a_{m+n-1}\right) } \\
= & \sum_{\sigma \in S_{n, m-1}} \operatorname{sgn}(\sigma) \alpha\left(\beta\left(a_{\sigma(1)}, \ldots, a_{\sigma(n)}\right), a_{\sigma(n+1)}, \ldots, a_{\sigma(n+m-1)}\right) \\
& -(-1)^{(m-1)(n-1)} \sum_{\sigma \in S_{m, n-1}} \operatorname{sgn}(\sigma) \beta\left(\alpha\left(a_{\sigma(1)}, \ldots, a_{\sigma(m)}\right), a_{\sigma(m+1)}, \ldots, a_{\sigma(m+n-1)}\right) .
\end{aligned}
$$

Being a bi-derivation, any Poisson bracket on $A$ is of the form

$$
\{a, b\}=\pi(a, b), \quad a, b \in A,
$$

for a unique $\pi \in \mathfrak{X}^{2}(A)$, and the condition $\operatorname{char}(k) \neq 2$ implies that the Jacobi identity of $\{-,-\}$ is equivalent to $[\pi, \pi]=0$.

Notation 3.2. For any integer $r \geq 1$, let

$$
S_{[2 r]}=\left\{\sigma \in S_{2 r}: \sigma(1)<\sigma(3)<\ldots<\sigma(2 r-1) \text { and } \sigma(2 i-1)<\sigma(2 i), i=1, \ldots, r\right\},
$$

and for $\pi \in \mathfrak{X}^{2}(A)$, set $\pi^{[r]} \in \mathfrak{X}^{2 r}(A)$ by

$$
\pi^{[r]}\left(a_{1}, \ldots, a_{2 r}\right)=\sum_{\sigma \in S_{[2 r]}}(\operatorname{sgn} \sigma) \pi\left(a_{\sigma(1)}, a_{\sigma(2)}\right) \ldots \pi\left(a_{\sigma(2 r-1)}, a_{\sigma(2 r)}\right) .
$$

Lemma 3.3. For any $\pi \in \mathfrak{X}^{2}(A)$ and any integer $r \geq 1$, one has $\pi^{\wedge r}=r ! \pi^{[r]}$.

Proof. Denote by $R_{2}^{r}$ the set of all elements $\sigma \in S_{2 r}$ such that $\sigma(2 i-1)<\sigma(2 i)$ for all $i=1, \ldots, r$. In particular, $R_{2}^{2}$ is just the set of all $(2,2)$-shuffles. By definition, for any $a_{1}, \ldots, a_{2 r} \in A$, one has

$$
\pi^{\wedge r}\left(a_{1}, \ldots, a_{2 r}\right)=\sum_{\sigma \in R_{2}^{r}}(\operatorname{sgn} \sigma) \pi\left(a_{\sigma(1)}, a_{\sigma(2)}\right) \ldots \pi\left(a_{\sigma(2 r-1)}, a_{\sigma(2 r)}\right) .
$$

For each $\sigma \in R_{2}^{r}$, we can reorder the factors in the right hand side of the above expression so we may assume that $\sigma(1)<\sigma(3)<\ldots<\sigma(2 r-1)$, i.e., $\sigma \in S_{[2 r]}$. Thus

$$
\begin{aligned}
\pi^{\wedge r}\left(a_{1}, \ldots, a_{2 r}\right) & =r ! \sum_{\sigma \in S_{[2 r]}}(\operatorname{sgn} \sigma) \pi\left(a_{\sigma(1)}, a_{\sigma(2)}\right) \ldots \pi\left(a_{\sigma(2 r-1)}, a_{\sigma(2 r)}\right) \\
& =r ! \pi^{[r]}\left(a_{1}, \ldots, 2_{2 r}\right) .
\end{aligned}
$$

\section{Q.E.D.}

Definition 3.4. The rank of a Poisson structure $\pi$ on $A$, denoted by $\operatorname{rank}(\pi)$, is defined to be $2 r$, where $r$ is the largest integer such that $\pi^{[r]} \neq 0$.

Let $A$ be a finitely generated $k$-algebra and let $\mathfrak{m}$ be a maximal ideal of $A$. Recall that $\mathfrak{m} / \mathfrak{m}^{2}$ is a vector space over the field $A / \mathfrak{m}$. As $A$ is finitely generated and $k$ is algebraically closed, $A / \mathfrak{m} \cong k$ [1, Corollary 7.10]. Let $\alpha \in \mathfrak{X}^{n}(A)$ and $a_{1}, \ldots, a_{n} \in A$. Suppose $a_{i} \in \mathfrak{m}^{2}$ for some $i$. Then by the Leibniz rule, one see that $\alpha\left(a_{1}, \ldots, a_{n}\right)$ lies in $\mathfrak{m}$. Therefore, $\alpha$ induces an alternating $k$-linear map

$$
\alpha_{\mathfrak{m}}: \wedge^{n}\left(\mathfrak{m} / \mathfrak{m}^{2}\right) \rightarrow A / \mathfrak{m}
$$


Assume now that $A$ is a Poisson $k$-algebra with Poisson structure $\pi \in \mathfrak{X}^{2}(A)$. Then $\pi$ induces an alternating $k$-linear map for each maximal ideal $\mathfrak{m}$ of $A$

$$
\pi_{\mathfrak{m}}:\left(\mathfrak{m} / \mathfrak{m}^{2}\right) \wedge\left(\mathfrak{m} / \mathfrak{m}^{2}\right) \rightarrow k .
$$

Consequently, one has the skew-symmetric linear transformation

$$
\pi_{\mathfrak{m}}^{\sharp}: \mathfrak{m} / \mathfrak{m}^{2} \rightarrow\left(\mathfrak{m} / \mathfrak{m}^{2}\right)^{*} .
$$

of $k$-vector spaces for each maximal ideal $\mathfrak{m}$ of $A$.

Definition 3.5. The rank of a Poisson structure $\pi$ at a maximal ideal $\mathfrak{m}$ of $A$, denoted by $\operatorname{rank}\left(\pi_{\mathfrak{m}}\right)$, is defined to be the rank of $\pi_{\mathfrak{m}}^{\sharp}$. It is an even integer as we are assuming that $\operatorname{char}(k) \neq$ 2 .

Let $V$ be a $k$-vector space and let $\omega \in \wedge^{2} V$. Similar to the case of multi-derivation, we have the element $\omega^{[l]} \in \wedge^{2 l} V$. Following previous notation, define $\omega^{[l]} \in \wedge^{2 l} V$ by

$$
\omega^{[l]}\left(v_{1}, \ldots, v_{2 l}\right)=\sum_{\sigma \in S_{[2 l]}}(\operatorname{sgn} \sigma) \omega\left(v_{\sigma(1)}, v_{\sigma(2)}\right) \ldots \omega\left(v_{\sigma(2 l-1)}, v_{\sigma(2 l)}\right), \quad v_{i} \in V^{*} .
$$

Remark 3.6. It is easy to see that $\left(\pi^{[l]}\right)_{\mathfrak{m}}=\left(\pi_{\mathfrak{m}}\right)^{[l]} \in \wedge^{2 l}\left(\mathfrak{m} / \mathfrak{m}^{2}\right)^{*}$. Therefore there is no ambiguity to write $\pi_{m}^{[l]}$.

Proposition 3.7. Let $\pi$ be a Poisson structure on $A$. Then $\operatorname{rank}(\pi) \geq \operatorname{rank}\left(\pi_{\mathfrak{m}}\right)$ for every maximal ideal $\mathfrak{m}$. Moreover, if $A$ is reduced, then there exists some maximal ideal $\mathfrak{m}$ such that $\operatorname{rank}(\pi)=\operatorname{rank}\left(\pi_{\mathfrak{m}}\right)$.

Proof. Suppose $\operatorname{rank}(\pi)=2 r$. Then $\pi^{[r+1]}=0$, hence $\pi_{\mathfrak{m}}^{[r+1]}=0$, which implies that $\operatorname{rank}\left(\pi_{\mathfrak{m}}\right) \leq$ $2 r$.

To prove the second part, we assume the contrary. Suppose $\operatorname{rank}\left(\pi_{\mathfrak{m}}\right)<2 r$ for every maximal ideal $\mathfrak{m}$, so that $\pi_{\mathfrak{m}}^{[r]}=0$. By definition, it means that $\pi_{\mathfrak{m}}^{[r]}\left(b_{1}, \ldots, b_{2 r}\right)=0$ for all $b_{i} \in \mathfrak{m}$. In other words, one has $\pi^{[r]}\left(b_{1}, \ldots, b_{2 r}\right) \in \mathfrak{m}$ for all $b_{i} \in \mathfrak{m}$. Fix any maximal ideal $\mathfrak{m}$, one has $A=k \bigoplus \mathfrak{m}$. Let $a_{1}, \ldots, a_{2 r} \in A$ and write $a_{i}=c_{i}+b_{i}$, where $c_{i} \in k$ and $b_{i} \in \mathfrak{m}$. Then

$$
\pi^{[r]}\left(a_{1}, \ldots, a_{2 r}\right)=\pi^{[r]}\left(c_{1}+b_{1}, \ldots, c_{2 r}+b_{2 r}\right)=\pi^{[r]}\left(b_{1}, \ldots, b_{2 r}\right) \in \mathfrak{m} .
$$

This holds for every maximal ideal $\mathfrak{m}$. Therefore $\pi^{[r]}\left(a_{1}, \ldots, a_{2 r}\right)$ lies in the Jacobson radical of $A$. Since $A$ is a finitely generated $k$-algebra, its Jacobson radical equals its nil-radical, which is zero by our assumption that $A$ is reduced. So $\pi^{[r]}=0$, contradicting that the rank of $\pi$ is $2 r$.

\section{Q.E.D.}

Example 3.8. Let $A=k[x, y, z] / I$, where $I=\left\langle x^{2}\right\rangle$. Then $A$ is not reduced since $x$ is a nilpotent element. Let $\pi \in \mathfrak{X}^{2}(A)$ be defined by

$$
\pi(x, y)=x, \pi(x, z)=x, \pi(y, z)=0 .
$$

Then for any $f \in k[x, y, z]$, one has $\pi(x, f) \in\langle x\rangle$. Therefore $\pi$ is well-defined because $\pi\left(x^{2}, f\right)=$ $2 x \pi(x, f) \in I$. Since $\pi \neq 0$, one must have $\operatorname{rank}(\pi)>0$. Actually it can be easily seen that 
$\operatorname{rank}(\pi)=2$. On the other hand, maximal ideals of $A$ are of the form $\mathfrak{m}=\left\langle x, y-y_{0}, z-z_{0}\right\rangle$, where $y_{0}, z_{0} \in k$. Fix arbitrary $y_{0}$ and $z_{0}$. Then $\pi_{m}$ is given by

$$
\begin{aligned}
& \pi_{m}\left(x, y-y_{0}\right)=\pi\left(x, y-y_{0}\right)=\pi(x, y)=x \in m, \\
& \pi_{m}\left(x, z-z_{0}\right)=\pi\left(x, z-z_{0}\right)=\pi(x, z)=x \in m, \\
& \pi_{m}\left(y-y_{0}, z-z_{0}\right)=\pi(y, z)=0 .
\end{aligned}
$$

Hence $\pi_{\mathfrak{m}}$ is trivial, implying that $\operatorname{rank}\left(\pi_{\mathfrak{m}}\right)=0$, for any maximal ideals of $A$.

3.2. Frobenius Poisson $\mathbb{T}$-Pfaffians. Recall that an algebraic torus $\mathbb{T}$ over $k$ is an algebraic group isomorphic to $\left(k^{*}\right)^{n}$ for some non-negative integer $n$. A character of $\mathbb{T}$ is an algebraic group homomorphism $\lambda: \mathbb{T} \rightarrow k^{*}$. The set of all characters of $\mathbb{T}$ forms an abelian group with the group operation being pointwise multiplication and is denoted by $X^{*}(\mathbb{T})$, called the character group of $\mathbb{T}$. Any identification $\mathbb{T} \cong\left(k^{*}\right)^{n}$ gives coordinates $\left(t_{1}, \ldots, t_{n}\right)$ on $\mathbb{T}$. Let $\lambda \in X^{*}(\mathbb{T})$. Then $\lambda$ is a morphism of varieties, so $\lambda\left(t_{1}, \ldots, t_{n}\right)$ should be a Laurent monomial in these variables. As $\lambda$ is also a group homomorphism, $\lambda$ should be a Laurent monomial. Hence

$$
\lambda\left(t_{1}, \ldots, t_{n}\right)=t_{1}^{r_{1}} \ldots t_{n}^{r_{n}}
$$

for some $r_{1}, \ldots, r_{n} \in \mathbb{Z}$. On the other hand, it can be easily seen that all $\lambda$ of the form above are characters of $\mathbb{T}$. One thus sees that $X^{*}(\mathbb{T})$ is isomorphic to $\mathbb{Z}^{n}$, a free abelian group of rank $n$. For the rest of the chapter, we will identify $X^{*}(\mathbb{T})$ with $\mathbb{Z}^{n}$. Also, we write $t^{\lambda}$ for $\lambda(t)$.

Suppose $\lambda \in X^{*}(\mathbb{T})$, then it induces a map $\lambda: \mathfrak{t} \rightarrow k$, where $\mathfrak{t}$ is the Lie algebra of $\mathbb{T}$, given by

$$
\lambda(h)=\sum_{i=1}^{n} \lambda_{i}\left(h_{i}\right),
$$

where $\lambda=\left(\lambda_{1}, \ldots, \lambda_{n}\right)$ and $h=\left(h_{1}, \ldots, h_{n}\right)$.

Suppose now $\mathbb{T}$ acts on a vector space $V$. Let $v \in V$. If there exists some function $\lambda: \mathbb{T} \rightarrow k^{*}$ such that $t v=\lambda(t) v$ for all $t \in \mathbb{T}$, we say that $v$ is a weight vector with weight $\lambda$. The set of all $\lambda$-weight vectors form a vector subspace of $V$, called the weight space and is denoted by $V_{\lambda}$.

Definition 3.9. Let $A$ be a $k$-algebra with a $\mathbb{T}$-action. The action is said to be rational if $A$ can be decomposed into a direct sum of weight spaces, i.e.

$$
A=\bigoplus_{\lambda \in X^{*}(T)} V_{\lambda}
$$

Note that the coordinate ring of an affine $\mathbb{T}$-variety over $k$ is an algebra with a rational $\mathbb{T}$-action.

Definition 3.10. Suppose $\mathbb{T}$ acts on $A$ rationally. Let $a \in A$. From the weight-space decomposition of $A$, we can write $a=\sum_{i=1}^{n} a_{i}$, where $a_{i} \in V_{\lambda_{i}}$ for some $\lambda_{i} \in X^{*}(\mathbb{T})$. Let $h \in \mathfrak{t}$, the $k$-derivation on $A$ generated by $h$, denoted by $\partial_{h}$, is defined as

$$
\partial_{h}(a)=\sum_{i=1}^{n} \lambda_{i}(h) a_{i} .
$$

Definition 3.11. Let $A$ be a Poisson $k$-algebra. A Poisson $\mathbb{T}$-action on $A$ is a $\mathbb{T}$-action on $A$ by Poisson automorphisms. A rational Poisson $\mathbb{T}$-action is a rational $\mathbb{T}$-action on $A$ which is at the same time a Poisson $\mathbb{T}$-action. 
Lemma 3.12. For $h \in \mathfrak{t}$, the derivation $\partial_{h}$ induced from a rational Poisson torus action is a Poisson derivation.

Proof. Let $a=\sum_{i=1}^{n} a_{i}$ and $b=\sum_{j=1}^{m} b_{i}$. One has $\left\{a_{i}, b_{j}\right\} \in V_{\lambda_{i}+\mu_{j}}$, therefore

$$
\partial_{h}\{a, b\}=\partial_{h}\left(\sum_{i, j}\left\{a_{i}, b_{j}\right\}\right)=\sum_{i, j}\left(\lambda_{i}+\mu_{j}\right)(h)\left\{a_{i}, b_{j}\right\} .
$$

On the other hand,

$$
\begin{aligned}
& \left\{\partial_{h}(a), b\right\}+\left\{a, \partial_{h}(b)\right\} \\
= & \left\{\sum_{i} \lambda_{i}(h) a_{i}, \sum_{j} b_{j}\right\}+\left\{\sum_{i} a_{i}, \sum_{j} \mu_{j}(h) b_{j}\right\} \\
= & \sum_{i, j} \lambda_{i}(h)\left\{a_{i}, b_{j}\right\}+\sum_{i, j} \mu_{j}(h)\left\{a_{i}, b_{j}\right\} \\
= & \sum_{i, j}\left(\lambda_{i}+\mu_{j}\right)(h)\left\{a_{i}, b_{j}\right\} .
\end{aligned}
$$

Q.E.D.

Definition 3.13. Let $(A, \pi)$ be a finitely generated Poisson algebra with a rational Poisson $\mathbb{T}$-action. Suppose the rank of the Poisson structure on $A$ is $2 r$. A Poisson $\mathbb{T}$-Pfaffian of $(A, \pi)$ is a non-zero element $\sigma \in \mathfrak{X}^{\text {top }}(A)$ of the form

$$
\sigma=\pi^{[r]} \wedge \partial_{h_{1}} \wedge \ldots \wedge \partial_{h_{l}}
$$

where $h_{1}, \ldots, h_{l} \in \mathfrak{t}$.

For $a \in A$, define $X_{a} \in \mathfrak{X}^{1}(A)$ by $X_{a}(b)=\{a, b\}$ for $b \in A$. It is easy to see that $X_{a}$ is a Poisson derivation of $A$. We will refer to $X_{a}$ as the Hamiltonian derivation of $a$.

Proposition 3.14. The following formula holds for all positive integers $l$ and all $a_{1}, \ldots, a_{2 l} \in A$ :

$$
\pi^{[l]}\left(a_{1}, \ldots, a_{2 l}\right)=-\left(\pi^{[l-1]} \wedge X_{a_{2 l}}\right)\left(a_{1}, \ldots, a_{2 l-1}\right) .
$$

In particular, $\pi^{[r]} \wedge X_{a}=0$ for the Hamiltonian derivation $X_{a}$, where $r$ is the half rank of $\pi$.

Proof. By definition, one has

$$
\begin{aligned}
& \left(\pi^{[l-1]} \wedge X_{a_{2 l}}\right)\left(a_{1}, \ldots, a_{2 l-1}\right) \\
= & \sum_{\sigma \in S_{2 l-2,1}}(\operatorname{sgn} \sigma) \pi^{[l-1]}\left(a_{\sigma(1)}, \ldots, a_{\sigma(2 l-2)}\right) X_{a_{2 l}}\left(a_{\sigma(2 l-1)}\right) \\
= & -\sum_{\sigma \in S_{2 l-2,1}}(\operatorname{sgn} \sigma) \pi^{[l-1]}\left(a_{\sigma(1)}, \ldots, a_{\sigma(2 l-2)}\right) \pi\left(a_{\sigma(2 l-1)}, a_{2 l}\right) \\
= & -\sum_{\substack{\sigma \in S_{2 l-2,2} \\
\sigma(2 l)=2 l}}(\operatorname{sgn} \sigma) \pi^{[l-1]}\left(a_{\sigma(1)}, \ldots, a_{\sigma(2 l-2)}\right) \pi\left(a_{\sigma(2 l-1)}, a_{\sigma(2 l)}\right) .
\end{aligned}
$$

For each $\sigma \in S_{2 l-2,2}$ with $\sigma(2 l)=2 l$, we know that

$$
\begin{aligned}
& (\operatorname{sgn} \sigma) \pi^{[l-1]}\left(a_{\sigma(1)}, \ldots, a_{\sigma(2 l-2)}\right) \pi\left(a_{\sigma(2 l-1)}, a_{\sigma(2 l)}\right) \\
= & \sum_{\tau} \operatorname{sgn}(\tau \sigma) \pi\left(a_{\tau \sigma(1)}, a_{\tau \sigma(2)}\right) \ldots \pi\left(a_{\tau \sigma(2 l-3)}, a_{\tau \sigma(2 l-2)}\right) \pi\left(a_{\sigma(2 l-1)}, a_{\sigma(2 l)}\right),
\end{aligned}
$$


where $\tau$ runs through all permutations on $\{\sigma(1), \ldots, \sigma(2 l-2)\}$ such that $\tau \sigma(1)<\tau \sigma(3)<\ldots<$ $\tau \sigma(2 l-3)$ and $\tau \sigma(2 i-1)<\tau \sigma(2 i)$ for $i=1, \ldots, l-1$. If we extend each $\tau$ to a permutation in $S_{2 l}$ by setting $\tau \sigma(2 l-1)=\sigma(2 l-1)$ and $\tau \sigma(2 l)=\sigma(2 l)=2 l$, then

$$
\left(\pi^{[l-1]} \wedge X_{a_{2 l}}\right)\left(a_{1}, \ldots, a_{2 l-1}\right)=-\sum_{\tau \sigma} \operatorname{sgn}(\tau \sigma) \pi\left(a_{\tau \sigma(1)}, a_{\tau \sigma(2)}\right) \ldots \pi\left(a_{\tau \sigma(2 l-1)}, a_{\tau \sigma(2 l)}\right),
$$

where $\tau \sigma$ runs through all permutations in $R_{2}^{l}$ such that $\tau \sigma(1)<\tau \sigma(3)<\ldots<\tau \sigma(2 l-3)$ and $\tau \sigma(2 l)=2 l$. Recall that $S_{[2 r]}$ is the subset of permutations in $R_{2}^{l}$ such that $\sigma(1)<\sigma(3) \ldots<$ $\sigma(2 l-1)$. By rearranging the order of the terms, we obtain

$$
\left(\pi^{[l-1]} \wedge X_{a_{2 l}}\right)\left(a_{1}, \ldots, a_{2 l-1}\right)=\sum_{\sigma \in S_{[2 r]}}(\operatorname{sgn} \sigma) \pi\left(a_{\sigma(1)}, a_{\sigma(2)}\right) \ldots \pi\left(a_{\sigma(2 l-1)}, a_{\sigma(2 l)}\right) .
$$

On the other hand, still by definition

$$
\pi^{[l]}\left(a_{1}, \ldots, a_{2 l}\right)=\sum_{\sigma \in S_{[2 r]}}(\operatorname{sgn} \sigma) \pi\left(a_{\sigma(1)}, a_{\sigma(2)}\right) \ldots \pi\left(a_{\sigma(2 l-1)}, a_{\sigma(2 l)}\right) .
$$

Therefore,

$$
\pi^{[l]}\left(a_{1}, \ldots, a_{2 l}\right)=-\pi^{[l-1]} \wedge X_{a_{2 l}}\left(a_{1}, \ldots, a_{2 l-1}\right)
$$

Q.E.D.

Proposition 3.15. Let $\sigma$ be a Poisson $\mathbb{T}$-Pfaffian and $V$ a Hamiltonian derivation or a derivation generated by some element of the Lie algebra of $\mathbb{T}$ for the torus action. One has $[V, \sigma]=0$.

Proof. By definition,

$$
[V, \sigma]=[V, \pi] \wedge \pi^{[r-1]} \wedge \partial_{h_{1}} \wedge \ldots \wedge \partial_{h_{l}}+\sum_{i=1}^{l} \pi^{[r]} \wedge \partial_{h_{1}} \wedge \ldots \wedge\left[V, \partial_{h_{i}}\right] \wedge \ldots \wedge \partial_{h_{l}} .
$$

If $V$ is Hamiltonian, then $[V, \pi]$ is zero. On the other hand, $V$ is Hamiltonian implies $\left[V, \partial_{h_{i}}\right]$ is Hamiltonian. Then $\pi^{[r]} \wedge\left[V, \partial_{h_{i}}\right]$ is also zero by Proposition 3.14, If $V$ is generated by some element of the Lie algebra of $\mathbb{T}$ for the torus action, then both $[V, \pi]$ and $\left[V, \partial_{h_{i}}\right]$ are zero.

\section{Q.E.D.}

Remark 3.16. It is straightforward to see that the results in this section generalize to $\mathbb{T}$-Poisson varieties.

3.3. Rank of Poisson CGL extensions. We first recall from [9, Section 1.3] the definition of Poisson CGL extensions.

Definition 3.17. A Poisson CGL extension is a polynomial algebra $k\left[x_{1}, \ldots, x_{n}\right]$ with a $\mathbb{T}$ Poisson structure for which $x_{1}, \ldots, x_{n}$ are $\mathbb{T}$-eigenvectors, where $\mathbb{T}$ is an algebraic torus, together with elements $h_{1}, \ldots, h_{n} \in \mathfrak{t}$ such that the following conditions are satisfied for $1 \leq i \leq n$ :

(a) For $i<j,\left\{x_{i}, x_{j}\right\}=\lambda_{j}\left(h_{i}\right) x_{i} x_{j}+\delta_{i}\left(x_{j}\right)$ for some $\lambda_{i} \in \mathfrak{t}^{*}$ and some locally nilpotent derivation $\delta_{i}$ on $k\left[x_{i+1}, \ldots, x_{n}\right]$ with $\delta_{i}\left(x_{j}\right) \in k\left[x_{i+1}, \ldots, x_{j-1}\right]$.

(b) The $h_{i}$-eigenvalue of $x_{i}$ is non-zero, i.e. $\lambda_{i}\left(h_{i}\right) \neq 0$. 
Remark 3.18. A Poisson CGL extension is essentially an affine space equipped with a special $\mathbb{T}$-Poisson structure. Thus the notions of Poisson $\mathbb{T}$-Pfaffians and Frobenius Poisson T-Pfaffians make sense for Poisson CGL extensions. In this case, the vector field $\partial_{h}$ generated by an element $h \in \mathfrak{t}$ is given by

$$
\partial_{h}=\lambda_{1}(h) x_{1} \partial_{1}+\lambda_{2}(h) x_{2} \partial_{2}+\ldots+\lambda_{n}(h) x_{n} \partial_{n} .
$$

Definition 3.19. The Poisson bivector of a Poisson CGL extension, which is written as

$$
\pi=\sum_{i<j}\left(\lambda_{j}\left(h_{i}\right) x_{i} x_{j}+\delta_{i}\left(x_{j}\right)\right) \partial_{i} \wedge \partial_{j},
$$

is called a Poisson CGL bivector field.

Remark 3.20. The bivector field defined by

$$
\pi_{0}=\sum_{i<j} \lambda_{j}\left(h_{i}\right) x_{i} x_{j} \partial_{i} \wedge \partial_{j}
$$

is a Poisson bivector field, which is called the log-canonical part of $\pi$. The bivector field $\sum_{i<j}\left(\delta_{i}\left(x_{j}\right)\right) \partial_{i} \wedge \partial_{j}$ is not necessarily a Poisson vector field, called the $\delta$-part of $\pi$.

Proposition 3.21. The rank of the Poisson bivector $\pi$ of a Poisson CGL extension is greater than or equal to that of $\pi_{0}$.

Proof. Suppose $\pi^{[r]}=0$ for some non-negative integer $r$. Let $w=\pi-\pi_{0}=\sum_{i<j} \delta_{i}\left(x_{j}\right) \partial_{i} \wedge \partial_{j}$. Then

$$
\pi^{[r]}=\pi_{0}^{[r]}+\sum_{l=0}^{r-1} \pi_{0}^{[l]} \wedge w^{[r-l]} .
$$

Let $1 \leq i_{1}<\ldots<i_{2 r} \leq n$. For $0 \leq l \leq r-1$, one has

$$
\begin{aligned}
& \pi_{0}^{[l]} \wedge w^{[r-l]}\left(d x_{i_{1}} \wedge \ldots \wedge d x_{i_{2 r}}\right) \\
= & \sum_{\sigma \in S_{2 l, 2 r-2 l}} \pi_{0}^{[j]}\left(d x_{i_{\sigma(1)}} \wedge \ldots \wedge d x_{i_{\sigma(2 l)}}\right) w^{[r-j]}\left(d x_{i_{\sigma(2 l+1)}} \wedge \ldots \wedge d x_{\left.i_{\sigma(2 r)}\right)}\right)
\end{aligned}
$$

For each $\sigma \in S_{2 l, 2 r-2 l}$, we know that $\pi_{0}^{[l]}\left(d x_{i_{\sigma(1)}} \wedge \ldots \wedge d x_{i_{\sigma(2 l)}}\right)$ is a scalar multiple of $x_{i_{\sigma(1)}} \ldots x_{i_{\sigma(2 l)}}$. However $w^{[r-l]}\left(d x_{i_{\sigma(2 l+1)}} \wedge \ldots \wedge d x_{i_{\sigma(2 r)}}\right)$ does not involve the variable $x_{i_{\sigma(2 r)}}$ since $\delta_{i}\left(x_{j}\right)$ does not involve the variable $x_{j}$ for every $i<j$. Therefore the coefficient of $x_{i_{1}} \ldots x_{i_{2 r}}$ in $\pi_{0}^{[l]} \wedge w^{[r-l]}\left(d x_{i_{1}} \wedge\right.$ $\left.\ldots \wedge d x_{i_{2 r}}\right)$ is zero. As $\pi_{0}\left(d x_{i_{1}} \wedge \ldots \wedge d x_{i_{2 r}}\right)$ is a scalar multiple of $x_{i_{1}} \ldots x_{i_{2 r}}$, our assumption implies that $\pi_{0}\left(d x_{i_{1}} \wedge \ldots \wedge d x_{i_{2 r}}\right)=0$ for every $1 \leq i_{1}<\ldots<i_{2 r} \leq n$. Hence $\pi_{0}^{[r]}=0$.

Q.E.D.

Let $\left(k\left[x_{1}, \ldots, x_{n}\right], \pi\right)$ be a Poisson CGL extension. Let $e_{i}=x_{i} \partial_{i}$ and $V$ be the $n$-dimensional $k$-vector space with basis $\left\{e_{1}, \ldots, e_{n}\right\}$. Then $\pi_{0}$, the log-canonical term of $\pi$, is represented by the skew-symmetric matrix $\Omega-\Omega^{T}$, where $\Omega$ is the $n \times n$ strictly upper triangular matrix with entries $\lambda_{i}\left(h_{j}\right)$ for $i<j$. Suppose the rank of the matrix $\pi_{0}$ is $2 r$, let $v_{1}, \ldots, v_{n-2 r}$ be $n-2 r$ elements in $V$. Note that with the basis $e_{i}$ 's, the $v_{i}$ 's can be regarded as size $n$ column vectors. One can define an $n \times(2 n-2 r)$-matrix $M$ as

$$
M=\left(\begin{array}{lllll}
\Omega-\Omega^{T} & v_{1} & v_{2} & \ldots & v_{n-2 r}
\end{array}\right) .
$$


Lemma 3.22. The element $\pi_{0}^{[r]} \wedge v_{1} \wedge \ldots \wedge v_{n-2 r} \in \wedge^{n} V$ is non-zero if and only if $M$ is of full rank.

Proof. Denote the columns of $\pi_{0}$ by $w_{1}, \ldots, w_{n}$. Since the rank of $\pi_{0}$ is $2 r$, without loss of generality, we may assume that the image of $\pi_{0}$ is $\operatorname{Span}\left\{w_{1}, \ldots, w_{2 r}\right\}$. Since $\pi_{0} \in \wedge^{2} i m\left(\pi_{0}\right)$, $\pi_{0}^{[r]}$ is a nonzero multiple of $w_{1} \wedge \ldots \wedge w_{2 r}$. Hence $\pi_{0}^{[r]} \wedge v_{1} \wedge \ldots \wedge v_{n-2 r} \neq 0$ if and only if $w_{1}, \ldots, w_{2 r}, v_{1}, \ldots, v_{n-2 r}$ are linearly independent, which is equivalent to saying that $M$ is of full rank.

\section{Q.E.D.}

Lemma 3.23. Suppose $\pi^{[r]} \wedge \partial_{h_{i_{1}}} \wedge \ldots \wedge \partial_{h_{i_{n-2 r}}}=f \partial_{1} \wedge \ldots \wedge \partial_{n}$ is non-zero for some integers $\left\{i_{1}, \ldots, i_{n-2 r}\right\} \subset[1, n]$, where $f \in k\left[x_{1}, \ldots, x_{n}\right]$. Let $g=c x_{1}^{t_{1}} \ldots x_{n}^{t_{n}}$ be a non-zero monomial term in $f$, where $c \in k$. Then either $\left(t_{1}, t_{2}, \ldots, t_{n}\right)=(1,1, \ldots, 1)$ or there exists some integer $s$ in $[1, n]$ such that $t_{s}=0$ and $t_{j} \leq 1$ for all $j>s$.

Proof. Following the notation in the proof of Proposition 3.21, write

$$
\pi^{[r]} \wedge \partial_{h_{i_{1}}} \wedge \ldots \wedge \partial_{h_{i_{n-2 r}}}=\sum_{l=0}^{r} \pi_{0}^{[l]} \wedge w^{[r-l]} \wedge \partial_{h_{i_{1}}} \wedge \ldots \wedge \partial_{h_{i_{n-2 r}}} .
$$

Then the pairing with $d x_{1} \wedge \ldots \wedge d x_{n}$ of each term in the expansion of the expression

$$
\pi_{0}^{[l]} \wedge w^{[r-l]} \wedge \partial_{h_{i_{1}}} \wedge \ldots \wedge \partial_{h_{i_{n-2 r}}}
$$

is equal to a scalar multiple of

$$
\left(\prod_{j=1}^{l} \lambda_{\sigma(2 j-1)}\left(h_{\sigma(2 j)}\right) x_{\sigma(2 j-1)} x_{\sigma(2 j)}\right)\left(\prod_{j=l+1}^{r} \delta_{\sigma(2 j-1)}\left(x_{\sigma(2 j)}\right)\right)\left(\prod_{j=1}^{n-2 r} \lambda_{\sigma(2 r+j)}\left(h_{i_{j}}\right) x_{\sigma(2 r+j)}\right)
$$

for some $\sigma \in S_{n}$ such that $\sigma(2 j-1)<\sigma(2 j)$ for $j=l+1, l+2, \ldots, r$. To prove the statement, it suffices to prove that when $g=c x_{1}^{t_{1}} \ldots x_{n}^{t_{n}}$ is a non-zero monomial term in the product above, where $c \in k$, then either $\left(t_{1}, t_{2}, \ldots, t_{n}\right)=(1,1, \ldots, 1)$ or there exists some integer $s$ in $[1, n]$ such that $t_{s}=0$ and $t_{j} \leq 1$ for all $j>s$.

In the case when $l=r$, it is obvious $t_{1}=\ldots=t_{n}=1$. In the case when $l<r$, choose $j$ in $l+1, l+2, \ldots, r$ so that $\sigma(2 j)$ is maximal among $\sigma(2 l+2), \sigma(2 l+4), \ldots, \sigma(2 r)$. Let $s$ be $\sigma(2 j)$. Then in the second bracket in expression [3.1, the degrees of $x_{s}, x_{s+1}, \ldots, x_{n}$ are all zero. In the product of the first and the third bracket in expression 3.1, the degree of $x_{s}$ is zero and the degrees of $x_{s+1}, \ldots, x_{n}$ are at most one.

\section{Q.E.D.}

3.4. Proof of Theorem C. For the convenience of the readers, we first restate the Theorem C.

Theorem 3.24. If the rank of a Poisson CGL bivector field $\pi_{X}$ is equal to that of $\left(\pi_{X}\right)_{0}$, the log-canonical part of $\pi_{X}$, then a Frobenius Poisson $\mathbb{T}$-Pfaffian exists. 
Proof. Suppose $\pi^{[r]} \wedge \partial_{h_{i_{1}}} \wedge \ldots \wedge \partial_{h_{i_{n-2 r}}}=f \partial_{1} \wedge \ldots \wedge \partial_{n}$ is non-zero. Then by [2, Theorem 1.3.8], a non-zero scalar multiple of it defines a Frobenius splitting if and only if the coefficient of $\left(x_{1} \ldots x_{n}\right)^{p-1}$ in $f^{p-1}$ is a non-zero constant. By Lemma 3.23, we know that the coefficient of $\left(x_{1} \ldots x_{n}\right)^{p-1}$ in $f^{p-1}$ is equal to the $p-1$ power of the coefficient of $x_{1} \ldots x_{n}$ in $f$. Write $\pi_{0}^{[r]} \wedge \partial_{h_{i_{1}}} \wedge \ldots \wedge \partial_{h_{i_{n-2 r}}}=g \partial_{1} \wedge \ldots \wedge \partial_{n}$. From Proposition 3.21 we see that the coefficient of $x_{j_{1}} \ldots x_{j_{2 r}} \partial_{j_{1}} \wedge \ldots \wedge \partial_{j_{2 r}}$ in $\pi^{[r]}$ is equal to that in $\pi_{0}^{[r]}$ for all $\left\{j_{1}, \ldots, j_{2 r}\right\}$. Therefore the coefficient of $x_{1} \ldots x_{n}$ in $f$ is equal to that in $g$. But $g$ is just a scalar multiple of $x_{1} \ldots x_{n}$. So to prove the statement, we only need to prove that $\pi_{0}^{[r]} \wedge \partial_{h_{i_{1}}} \wedge \ldots \wedge \partial_{h_{i_{n-2}}}$ is zero.

We know that $\partial_{h_{j}}$ is represented by the size $n$ column vector with the $i$-th entry being $\lambda_{i}\left(h_{j}\right)$. Denote the $n \times n$ strictly upper triangular matrix with $i, j$-th entry being $\lambda_{i}\left(h_{j}\right)$, where $i<j$, by $\Omega$. Denote the $n \times n$ strictly lower triangular matrix with $i, j$-th entry being $\lambda_{i}\left(h_{j}\right)$, where $i>j$, by $L$. Then the matrix representing $\left(\partial_{h_{1}} \ldots \partial_{h_{n}}\right)$ is written as $\Omega+L+D$, where $D$ is the non-singular diagonal matrix with entries $\lambda_{i}\left(h_{i}\right)$. Therefore, by Lemma 3.22 , to prove the statement, we only need to prove that the following $n \times 2 n$ matrix

$$
M=\left(\Omega-\Omega^{T} \quad \Omega+L+D\right)
$$

is of full rank. When $n=1$, the conclusion is trivial. Suppose the conclusion holds for a general $n$. For $n+1$, denote the size $n$ column vector with $i$-th entry $\lambda_{i}\left(h_{n+1}\right)$ by $v_{1}$, denote the size $n$ row vector with $i$-th entry $\lambda_{n+1}\left(h_{i}\right)$ by $v_{2}^{T}$. Then one has

$$
\Omega^{\prime}=\left(\begin{array}{cc}
\Omega & v_{1} \\
0 & 0
\end{array}\right), D^{\prime}=\left(\begin{array}{cc}
D & 0 \\
0 & \lambda_{n+1}\left(h_{n+1}\right)
\end{array}\right) \text { and } L^{\prime}=\left(\begin{array}{cc}
L & 0 \\
v_{2}^{T} & 0
\end{array}\right) .
$$

Therefore the $(n+1) \times(2 n+2)$ matrix $M^{\prime}$ is

$$
M^{\prime}=\left(\begin{array}{cccc}
\Omega-\Omega^{T} & v_{1} & \Omega+L+D & v_{1} \\
-v_{1}^{T} & 0 & v_{2}^{T} & \lambda_{n+1}\left(h_{n+1}\right)
\end{array}\right) .
$$

Denote the length $2 n$ row vector $\left(-v_{1}^{T} v_{2}^{T}\right)$ by $w$. If we move the $(n+1)$-th column of $M^{\prime}$ to the last, $M^{\prime}$ becomes

$$
\left(\begin{array}{ccc}
M & v_{1} & v_{1} \\
w & \lambda_{n+1}\left(h_{n+1}\right) & 0
\end{array}\right)
$$

Subtract the last column from the second last column gives,

$$
\left(\begin{array}{ccc}
M & 0 & v_{1} \\
w & \lambda_{n+1}\left(h_{n+1}\right) & 0
\end{array}\right)
$$

By definition we know that $\lambda_{n+1}\left(h_{n+1}\right) \neq 0$. Hence the last row of the matrix above cannot be a linear combination of the first $n$ rows since the second last entry is zero for each of the first $n$ rows. Therefore $M^{\prime}$ is also of full rank.

\section{Q.E.D.}

3.5. Proof of Theorem D. To prepare for the proof of Theorem D, we first cite the following Proposition 3.25 and prove the following Lemma 3.26 .

Proposition 3.25. [11] Let $X$ be a non-singular variety split by $\sigma \in H^{0}\left(X, \omega_{X}^{1-p}\right)$. Then for all compatibly split subvarieties $Y$, one has $Y \subset Z(\sigma)$. 
Lemma 3.26. Let $Y$ be a closed subvariety of a non-singular variety $X$ with ideal sheaf $\mathcal{I}_{Y}$. Let $V \in H^{0}\left(X, \mathcal{T}_{X}\right)$. Then $Y$ is invariant under $V$ if and only if $\mathcal{I}_{Y, p}$ is invariant under $V_{p}$ in $\mathcal{O}_{X, p}$ for every closed point $p$ of some open subset $\mathcal{U}$ of $X$.

Proof. It suffices to prove the statement for the affine case. Let $X=\operatorname{Spec}(A)$, where $A$ is a finitely generated $k$-algebra which is also an integral domain. Suppose $Y$ is given by an ideal $I$ of $A$. Suppose in addition that for every $a \in I,\left[V_{p}, a\right] \in I_{p}$ for all $p \in \mathcal{U}$. That means $\left[V_{p}, a\right]$ is zero in the quotient $A_{p} / I_{p} \cong(A / I)_{p}$. Since $\mathcal{U}$ is dense, $[V, a]$ actually vanishes in $A / I$, which means $[V, a] \in I$. The other direction is obvious.

\section{Q.E.D.}

Theorem 3.27. Let $X$ be a non-singular variety over $k, V \in H^{0}\left(X, \mathcal{T}_{X}\right)$ and $\sigma \in H^{0}\left(X, \omega_{X}^{-1}\right)$. Suppose $X$ is split by $\sigma^{p-1}$ and $[V, \sigma]=0$. Then all compatibly split subvarieties of $\sigma^{p-1}$ are invariant under $V$. Moreover, if the Frobenius splitting is defined by a Poisson $\mathbb{T}$-Pfaffian, then all compatibly split subvarieties are $\mathbb{T}$-Poisson subvarieties.

Proof. Let $D$ be a compatibly split divisor. We first prove that $D$ must be invariant under $V$. Since $D^{r e g}$, the smooth locus of $D$, is open in $D$, we only need to prove the statement locally on $D^{r e g}$. Let $p$ be a closed point of $D^{r e g}$. Choose local parameters $z_{1}, \ldots, z_{n}$ of $X$ such that $D$ is locally defined by $z_{1}=0$. Denote $\left\langle z_{1}\right\rangle$ by $I$. Then

$$
V=\sum_{i=1}^{n} g_{i} \frac{\partial}{\partial z_{i}}, \text { for some } g_{i} \in O_{X, p} \subset k\left[\left[z_{1}, \ldots, z_{n}\right]\right]
$$

and

$$
\sigma=f \frac{\partial}{\partial z_{1}} \wedge \ldots \wedge \frac{\partial}{\partial z_{n}} \text {, for some } f \in O_{X, p} \subset k\left[\left[z_{1}, \ldots, z_{n}\right]\right] .
$$

By Proposition 3.25, this implies that $f=t_{1}^{m} h$ for some $h$ and $m \geq 1$. Here we choose $m$ to be maximal. Since $\sigma$ defines a Frobenius splitting, $m$ cannot be a multiple of $p$. On the other hand, by direct calculation, $[V, \sigma]=0$ implies

$$
0=\sum_{i=1}^{n} \frac{\partial}{\partial z_{i}}\left(g_{i} f\right)=\sum_{i=1}^{n} \frac{\partial}{\partial z_{i}}\left(g_{i} z_{1}^{m} h\right)=m g_{1} h z_{1}^{m-1}+z_{1}^{m} \sum_{i=1}^{n} \frac{\partial}{\partial z_{i}}\left(g_{i} h\right) .
$$

Therefore $g_{1} h \in I$. Because $m$ is chosen to be maximal, it follows that $g_{1} \in I$. Let $l \in O_{X, p}$, then

$$
\left[V, z_{1} l\right]=\sum_{i=1}^{n} g_{i} \frac{\partial}{\partial z_{i}}\left(z_{1} l\right)=g_{1} l+\sum_{i=1}^{n} z_{1} g_{i} \frac{\partial l}{\partial z_{i}} \in I .
$$

Hence $D$ is invariant under $V$.

Next we prove the statement by induction on the dimension of $X$. The case where $\operatorname{dim}(X)=1$ follows from above. Suppose the statement holds for all $X$ with $\operatorname{dim}(X)<n$, we consider the case where $\operatorname{dim}(X)=n$. By our assumption, $X$ is split by a $p-1$ power. The compatibly split codimension 1 subvarieties are exactly the irreducible components of $Z(\sigma)$, say, $D_{1}, \ldots, D_{m}$. Let $Y$ be a compatibly split subvariety, then $Y \subset \cup D_{i}$. Let $Y_{i}$ be the scheme-theoretic intersection of $Y$ and $D_{i}^{r e g}$. Since $D_{i}$ and $Y$ are compatibly split, the splitting of $X$ restricts to a splitting of 
$D_{i}^{r e g}$, compatibly splitting $Y_{i}$. Since $\operatorname{dim}\left(D_{i}^{r e g}\right)<n$, by induction hypothesis, we conclude that $Y_{i}$ is invariant under $V$.

The ideal sheaf $\mathcal{I}_{Y}$ is the product of the $\mathcal{I}_{Y_{i}}$ 's. Let $f_{i}$ be a section of $\mathcal{I}_{Y_{i}}$. By the product rule one has $\left[V, f_{1} \ldots f_{m}\right]=\sum_{i=1}^{m} f_{1} \ldots \widehat{f}_{i} \ldots f_{m}\left[V, f_{i}\right]$. Therefore $Y$ is invariant under $V$.

To prove the second part of the theorem, suppose the splitting is defined by a Poisson $\mathbb{T}$ Pfaffian $\sigma$ and let $Y$ be a compatibly-split subvariety. Then $[V, \sigma]=0$ by Proposition 3.15 , where $V$ is any Hamiltonian vector field or generating vector field of the $\mathbb{T}$-action. Hence, by the first part of the theorem, $Y$ is invariant under all Hamiltonian vector fields or generating vector fields of the $\mathbb{T}$-action. But this just means $Y$ is a $\mathbb{T}$-Poisson subvariety.

\section{Q.E.D.}

\section{A Frobenius Splitting of $G / U$ via Poisson $T$-Pfaffian}

4.1. Frobenius Splitting of Bott-Samelson varieties via Poisson T-Pfaffians. Let $G$ be a simply-connected semi-simple algebraic group over an algebraically closed field $k$ of arbitrary characteristic. Let $\mathbf{u}=\left(s_{\alpha_{1}}, \ldots, s_{\alpha_{n}}\right)=\left(s_{1}, \ldots, s_{n}\right)$ be any word in $W=N_{G}(T) / T$, the Weyl group of $G$. Recall that the Bott-Samelson variety $Z_{\mathbf{u}}$ associated to the word $\mathbf{u}$ is defined to be the quotient space of $P_{\mathbf{u}}=P_{\alpha_{1}} \times \cdots \times P_{\alpha_{n}} \subset G^{n}$ by the $B^{n}$ action on $P_{\mathbf{u}}$ given by

$$
\left(p_{1}, \ldots, p_{n}\right) \cdot\left(b_{1}, \ldots, b_{n}\right)=\left(p_{1} b_{1}, b_{1}^{-1} p_{2} b_{2}, \ldots, b_{n-1}^{-1} p_{n} b_{n}\right)
$$

where $p_{i} \in P_{\alpha_{i}}$ and $b_{i} \in B$ for all $1 \leq i \leq n$. It is well known that $Z_{\mathbf{u}}$ is an $n$-dimensional non-singular projective variety [2, Section 2.2].

Recall that a sub-expression of a word $\mathbf{u}=\left(s_{1}, s_{2}, \ldots, s_{n}\right)$ is an element $\gamma \in\left\{e, s_{1}\right\} \times\left\{e, s_{2}\right\} \times$ $\ldots \times\left\{e, s_{n}\right\}$, where $e$ is the identity element in $W$. The set of all sub-expressions of $\mathbf{u}$ is denoted by $\Upsilon_{\mathbf{u}}$. Each $\gamma=\left(\gamma_{1}, \ldots, \gamma_{n}\right) \in \Upsilon_{\mathbf{u}}$ determines an open embedding to $Z_{\mathbf{u}}$ via

$$
\Phi^{\gamma}: \quad k^{n} \rightarrow Z_{\mathbf{u}}, \quad\left(z_{1}, \ldots, z_{n}\right) \mapsto\left[u_{-\gamma_{1}\left(\alpha_{1}\right)}\left(z_{1}\right) \dot{\gamma}_{1}, \ldots, u_{-\gamma_{n}\left(\alpha_{n}\right)}\left(z_{n}\right) \dot{\gamma}_{n}\right] .
$$

The image of $\Phi^{\gamma}$ will be denoted by $\mathcal{O}^{\gamma}$. The atlas $\left\{\left(\mathcal{O}^{\gamma}, \Phi^{\gamma}\right): \gamma \in \Upsilon_{\mathbf{u}}\right\}$ will be called the standard atlas on $Z_{\mathbf{u}}$.

There is a natural $T$-action on $Z_{\mathbf{u}}$ given by

$$
t \cdot\left[p_{1}, p_{2}, \ldots, p_{n}\right]=\left[t p_{1}, p_{2}, \ldots, p_{n}\right], \quad t \in T, p_{i} \in P_{\alpha_{i}}, 1 \leq i \leq n .
$$

Moreover, by direct computation we see that $\mathcal{O}^{\gamma}$ is $T$-invariant for each $\gamma \in \Upsilon_{\mathbf{u}}$ with

$$
t \cdot \Phi^{\gamma}\left(z_{1}, \ldots, z_{n}\right)=\Phi^{\gamma}\left(t^{-\gamma^{1}\left(\alpha_{1}\right)} z_{1}, \ldots, t^{-\gamma^{n}\left(\alpha_{n}\right)} z_{n}\right),
$$

where $\gamma^{i}:=\gamma_{1} \gamma_{2} \ldots \gamma_{i} \in W$ for $1 \leq i \leq n$.

For the case $k=\mathbb{C}$, recall from [7, Section 2.2] that the product Poisson structure $\pi_{\mathrm{st}}^{n}$ on $P_{\mathbf{u}}$ projects to a well-defined Poisson structure $\pi_{\mathbf{u}}$ on $Z_{\mathbf{u}}$, which is invariant under the $T$-action. Therefore, $\left(Z_{\mathbf{u}}, \pi_{\mathbf{u}}\right)$ is a $T$-Poisson variety. The following theorem is proved in [7, Theorem 4.14] [7, Theorem 5.12] for the case $k=\mathbb{C}$. 
Theorem 4.1. For any $\gamma=\left(\gamma_{1}, \ldots, \gamma_{n}\right) \in \Upsilon_{u}$, in the coordinates $\left(z_{1}, \ldots z_{n}\right)$ on the affine chart $\mathcal{O}^{\gamma}$ of $Z_{\mathbf{u}}$, the Poisson structure $\pi_{\mathbf{u}}$ is given by

$$
\left\{z_{i}, z_{k}\right\}=\left\{\begin{array}{ll}
\left\langle\gamma^{i}\left(\alpha_{i}\right), \gamma^{k}\left(\alpha_{k}\right)\right\rangle z_{i} z_{k}, & \text { if } \gamma_{i}=e \\
-\left\langle\gamma^{i}\left(\alpha_{i}\right), \gamma^{k}\left(\alpha_{k}\right)\right\rangle z_{i} z_{k}-\left\langle\alpha_{i}, \alpha_{i}\right\rangle \delta_{i}\left(z_{k}\right) & \text { if } \gamma_{i}=s_{i}
\end{array}, \quad 1 \leq i<k \leq n,\right.
$$

where $\delta_{i}$ is some vector field on $Z_{\left(s_{i+1}, \ldots, s_{n}\right)}$, and $\left(z_{i+1}, \ldots, z_{n}\right)$ are regarded as coordinates on $\mathcal{O}^{\left(\gamma_{i+1}, \ldots, \gamma_{n}\right)} \subset Z_{\left(s_{i+1}, \ldots, s_{n}\right)}$ in the expression $\delta_{i}\left(z_{k}\right)$ for $k \geq i+1$. Moreover, $\left(\mathbb{C}\left[\mathcal{O}^{\gamma}\right],\left.\pi_{\mathbf{u}}\right|_{\mathcal{O}^{\gamma}}\right)$ is a Poisson CGL extension for each $\gamma \in \Upsilon_{\mathbf{u}}$ with the $h_{i}$-eigenvalue of $z_{i}$ given by $\lambda_{i}\left(h_{i}\right)=\left\langle\alpha_{i}, \alpha_{i}\right\rangle$ if $\gamma_{i}=e$ and $\lambda_{i}\left(h_{i}\right)=-\left\langle\alpha_{i}, \alpha_{i}\right\rangle$ if $\gamma_{i}=s_{i}$.

Remark 4.2. As mentioned in [7, Section 1.2], the formula in Theorem 4.1] is algebraic and of integral coefficients.

From now on let $G$ be a simply-connected semi-simple algebraic group over an algebraically closed field $k$ of characteristic $p>3$, equipped with the standard Poisson structure defined in Definition 2.7. The Weyl group of $G$ is naturally isomorphic to the Weyl group of its complex counterpart. We want to point out that the proof of Proposition 2.2, which is carried out on the Lie algebra level, does not depend on the characteristic of the ground field and can be naturally adapted to the non-singular algebraic case. Therefore the product Poisson structure projects to a well-defined Poisson structure on Bott-Samelson varieties and $G / U$ in this positive characteristic case as well. By the same reasoning, Bott-Samelson varieties and $G / U$ over $k$ are $T$-Poisson varieties. See also [7, Remark 5.21] for reference.

Moreover, the computation of the local expression of the Poisson bracket in Theorem 4.1, which is again carried out at the Lie algebra level in the Chevalley basis, can naturally be adapted to the positive characteristic case via reduction modulo $p$. Therefore when $G$ is a simply-connected semi-simple algebraic group over an algebraically closed field of characteristic $p>3$, the Poisson bracket on $Z_{\mathbf{u}}$ in the charts defined by (4.1), where $\mathbf{u}$ is a word of the Weyl group of $G$, shares the same formula as the Poisson bracket on $Z_{\mathbf{u}}$, where $\mathbf{u}$ is regarded as a word of the Weyl group of the complex counterpart of $G$. Since $p>3,\left\langle\alpha_{i}, \alpha_{i}\right\rangle$ is non-zero modulo $p$. Hence in characteristic $p$, the Poisson structure on the chart $\mathcal{O}^{\gamma}$ is also a Poisson CGL extension for each $\gamma \in \Upsilon_{\mathbf{u}}$.

Theorem 4.3. A Frobenius Poisson T-Pfaffian exists on $\left(Z_{\mathbf{u}}, \pi_{\mathbf{u}}\right)$ and is unique up to scalar multiple. Moreover, it vanishes on all the sub-Bott-Samelson variety $Z_{\mathbf{u}[i]}$.

Proof. The existence and uniqueness follows from Proposition 3.24 and Theorem 4.1, In the standard atlas, the Poisson $T$-Pfaffian on the open subset $\mathcal{O}^{\mathbf{e}}$ of $Z_{\mathbf{u}}$ is expressed as a scalar multiple of

$$
z_{1} z_{2} \ldots z_{n} \frac{\partial}{\partial z_{1}} \wedge \ldots \wedge \frac{\partial}{\partial z_{n}}
$$

The last claim follows from the fact that $z_{i}$ is the local defining equation for the sub-BottSamelson variety $Z_{\mathbf{u}[i]}$ in $\mathcal{O}^{\mathbf{e}}$.

Q.E.D. 
Remark 4.4. As the Frobenius Poisson T-Pfaffian in Theorem 4.3 vanishes on all the sub-BottSamelson variety $Z_{\mathbf{u}[i]}$, it provides a choice of anti-canonical section in [2, Theorem 2.2.3].

4.2. Proof of Theorem E. To prepare for the proof of Theorem E, we first prove the following Theorem 4.5.

Theorem 4.5. A Frobenius Poisson T-Pfaffian exists on $\left(G / B, \pi_{G / B}\right)$ and is unique up to scalar multiple.

Proof. Recall from $\$ 2.3$ that $\left(G / B, \pi_{G / B}\right)$ is a $T$-Poisson variety. Let $w_{0}$ be the longest element of $W$. Given a reduced word $w_{0}=s_{1} s_{2} \ldots s_{n}$, we get a sequence of simple reflections $\mathbf{w}_{0}=$ $\left(s_{1}, s_{2}, \ldots, s_{n}\right)$. Let $\mu_{\mathbf{w}_{0}}$ be the morphism $Z_{\mathbf{w}_{0}} \rightarrow G / B$ defined by

$$
\mu_{\mathbf{w}_{0}}: Z_{\mathbf{w}_{0}} \rightarrow G / B, \quad\left[p_{1}, p_{2}, \ldots, p_{n}\right] \mapsto p_{1} p_{2} \ldots p_{n} . B .
$$

It is obvious that $\mu_{\mathbf{w}_{0}}$ is $T$-invariant under (4.2). Denote by $p_{\mathbf{w}_{0}}$ the projection $P_{\alpha_{1}} \times \ldots \times P_{\alpha_{n}} \rightarrow$ $Z_{\mathbf{w}_{0}}$. We know that $p_{\mathbf{w}_{0}}$ is surjective and Poisson. Since $\mu_{\mathbf{w}_{0}} \circ p_{\mathbf{w}_{0}}$ equals the composition of the multiplication map $P_{\alpha_{1}} \times \ldots \times P_{\alpha_{n}} \rightarrow G$ with the projection map $G \rightarrow G / B$, the map

$$
\mu_{\mathbf{w}_{0}} \circ p_{\mathbf{w}_{0}}:\left(P_{\alpha_{1}} \times \ldots \times P_{\alpha_{n}}, \pi_{\mathrm{st}}^{n}\right) \rightarrow\left(G / B, \pi_{G / B}\right)
$$

is Poisson. Therefore the morphism

$$
\mu_{\mathbf{w}_{0}}:\left(Z_{\mathbf{w}_{0}}, \pi_{\mathbf{w}_{0}}\right) \rightarrow\left(G / B, \pi_{G / B}\right)
$$

is Poisson. In addition, $\mathcal{O}^{\mathbf{e}} \subset Z_{\mathbf{w}_{0}}$ is $T$-invariant under (4.2) and $\mu_{\mathbf{w}_{0}}$ restricts to a $T$-equivariant isomorphism from $\mathcal{O}^{\mathbf{e}}$ to $B w_{0} B / B \cap B_{-} B / B \subset G / B$. It follows from Theorem 4.3 that a Frobenius Poisson $T$-Pfaffian exists on $G / B$ and is unique up to scalar multiple.

Q.E.D.

For the convenience of the readers, we restate Theorem E.

Theorem 4.6. There exists a unique (up to scalar multiples) Poisson $T$-Pfaffian $\sigma$ on $\left(G / U, \pi_{G / U}\right)$ and $\sigma^{p-1}$ induces a Frobenius splitting on $G / U$.

Proof. Let $w_{0}$ be the longest element of $W$. We know from Theorem 4.5 that a Frobenius Poisson $T$-Pfaffian exists for $\left(B w_{0} B / B, \pi_{G / B}\right)$. Suppose it is given by

$$
\sigma=\pi_{G / B}^{[r]} \wedge v_{x_{1}} \wedge \ldots \wedge v_{x_{n-2 r}}
$$

where $x_{1}, \ldots, x_{n} \in \mathfrak{t}$. Now let $\widetilde{x}_{i}=\left(x_{i}, 0\right) \in \mathfrak{t} \oplus \mathfrak{t}$. Suppose the rank of $T$ is $m$. For $i=1, \ldots, m$, let $y_{i}=\left(0, e_{i}\right) \in \mathfrak{t} \oplus \mathfrak{t}$, where $e_{i} \in k^{m}$ is 1 on the $i$-th spot and 0 elsewhere. Here we have made the identification between $k^{m}$ and $\mathfrak{t}$ via $\left(k^{\times}\right)^{m} \cong T$. Consider the section

$$
\widetilde{\sigma}=\pi_{G / U}^{[r]} \wedge v_{\widetilde{x}_{1}} \wedge \ldots \wedge v_{\widetilde{x}_{n-2 r}} \wedge v_{y_{1}} \wedge \ldots \wedge v_{y_{m}} \in H^{0}\left(G / U, \omega_{G / U}^{-1}\right) .
$$

Since $B w_{0} B / U$ is a $T$-invariant open subset of $G / U$, we may regard $\pi_{G / U}$ as a Poisson structure on $B w_{0} B / U$ and $v_{x}$ as a generating vector field for the $T$-action on $B w_{0} B / U$. Then the restriction of $\sigma_{1}$ on $B w_{0} B / U$ is given by the same expression as the corresponding restricted $\pi_{G / U}$ and $v$. 
Choose a reduced word for $w_{0}=s_{1} s_{2} \ldots s_{n}$ and get a sequence of simple reflections $\mathbf{w}_{0}=$ $\left(s_{1}, s_{2}, \ldots, s_{n}\right)$. In the toric coordinates $\left(\varphi_{\mathbf{w}_{0}}^{\mathbf{e}}\right)^{-1}$ on $\left(B w_{0} B / B \cap B-B / B\right)$ defined by

$$
\varphi_{\mathbf{w}_{0}}^{\mathbf{e}}:\left(k^{\times}\right)^{n} \rightarrow B w_{0} B / B \cap B_{-} B / B, \quad\left(z_{1}, \ldots, z_{n}\right) \mapsto\left(\mu_{\mathbf{w}_{0}} \circ \Phi^{\mathbf{e}}\right)\left(z_{1}, \ldots, z_{n}\right),
$$

where $\Phi^{\mathbf{e}}$ is defined in (4.1) and $\mu_{\mathbf{w}_{0}}$ is defined in (4.3), we know from the previous section that $\sigma$ in (4.4) is given by a scalar multiple of

$$
z_{1} \ldots z_{n} \frac{\partial}{\partial z_{1}} \wedge \ldots \wedge \frac{\partial}{\partial z_{n}}
$$

With respect to the diagonal action of $T$ on $T \times\left(B w_{0} B / B\right)$, the map

$$
\kappa_{\dot{w}_{0}}: B w_{0} B / U \rightarrow T \times\left(B w_{0} B / B\right)
$$

defined by (2.7) is a $T$-equivariant isomorphism. Then

$$
\kappa_{\dot{w}_{0}} \widetilde{\sigma}=\left(\kappa_{\dot{w}_{0}} \pi_{G / U}\right)^{[r]} \wedge v_{\widetilde{x}_{1}} \wedge \ldots \wedge v_{\widetilde{x}_{n-2 r}} \wedge v_{y_{1}} \wedge \ldots \wedge v_{y_{m}}
$$

Here $v$ represents the generating vector field for the $T$-action on $\left(B w_{0} B / B\right) \times T$.

Now consider the parametrization

$$
\left(k^{\times}\right)^{m} \times\left(k^{\times}\right)^{n} \rightarrow T \times\left(B w_{0} B / B \cap B_{-} B / B\right) .
$$

Denote the coordinates on $T$ by $t_{1}, \ldots, t_{m}$. It is obvious that $v_{y_{i}}=t_{i} \frac{\partial}{\partial t_{i}}$. Therefore

$$
v_{y_{1}} \wedge \ldots \wedge v_{y_{m}}=t_{1} \ldots t_{m} \frac{\partial}{\partial t_{1}} \wedge \ldots \wedge \frac{\partial}{\partial t_{m}}
$$

On the other hand, it follows from Proposition 2.13 that the expression of $\kappa_{\dot{w}_{0}} \pi_{G / U}$ must be of the form

$$
\pi_{G / B}+\sum_{i, j} f_{i j} \frac{\partial}{\partial z_{i}} \wedge \frac{\partial}{\partial t_{j}} .
$$

Since the $\pi_{G / B}$ terms are the only terms in $\kappa_{\dot{w}_{0}} \pi_{G / U}$ that do not involve $\frac{\partial}{\partial t_{j}}$, we must have

$$
\begin{aligned}
\kappa_{\dot{w}_{0}} \widetilde{\sigma} & =\pi_{G / B}^{[r]} \wedge v_{\widetilde{x}_{1}} \wedge \ldots \wedge v_{\widetilde{x}_{n-2 r}} \wedge v_{y_{1}} \wedge \ldots \wedge v_{y_{m}} \\
& =z_{1} \ldots z_{n} t_{1} \ldots t_{m} \frac{\partial}{\partial z_{1}} \wedge \ldots \wedge \frac{\partial}{\partial z_{n}} \wedge \frac{\partial}{\partial t_{1}} \wedge \ldots \wedge \frac{\partial}{\partial t_{m}} .
\end{aligned}
$$

Hence $\kappa_{\dot{w}_{0}} \tilde{\sigma}$ gives a Frobenius splitting on $\left(B w_{0} B / B\right) \times T$, which implies that $\tilde{\sigma}$ gives a Frobenius splitting on $B w_{0} B / U$ and hence on $G / U$.

Proposition 2.13 implies that $\kappa_{\dot{w}_{0}} \pi_{G / U}$ is log-canonical in the same parametrization as above. Now $\widetilde{\sigma}$ is a Poisson T-Pfaffian and uniqueness also follows from the the local expression of $\kappa_{\dot{w}_{0}} \tilde{\sigma}$.

\section{Q.E.D.}

\section{REFERENCES}

[1] M. Atiyah, I. MacDonald. Introduction to commutative algebra, Addison-Wesley-Longman, Boston, 1969.

[2] M. Brion and S.Kumar, Frobenius splitting methods in geometry and representation theory, Progress in Mathematics, 231. Birkhäuser Boston, Inc., Boston, MA, 2005.

[3] V. Chari and A. Pressley, A guide to quantum groups, Cambridge University press, Cambridge, 1994.

[4] C. Chevalley, Sur certains groupes simples, Tohoku Math. J., 7 (1955), no.1-2 , 14-66.

[5] V. Deodhar, On some geometric aspects of Bruhat orderings, I. A finer decomposition of Bruhat cells, Invent. Math. 79 (1985), no. 3, 499-511. 
[6] V.G. Drinfeld, On Poisson homogeneous spaces of Poisson-Lie groups, Theoret. Math. Phys., 95 (1993), no.2, 524-525.

[7] B. Elek and J.-H. Lu, Bott-Samelson varieties and Poisson-Ore extensions, to appear in Int. Math. Res. Not. IMRN, rnz127, https://doi.org/10.1093/imrn/rnz127; also available as arXiv:1601.00047.

[8] P. Etingof and O. Schiffmann, Lectures on quantum groups, 2nd edition, international press, Boston, 2002.

[9] K. Goodearl and M. Yakimov, Cluster algebras on nilpotent Poisson algebras, 2018, arXiv:1801.01963v2.

[10] J. Humphreys, Introduction to Lie algebras and representations theory, 3rd edition, Springer-Verlag, New York, 1980.

[11] S. Kumar and V.B. Mehta, Finiteness of the number of compatibly split subvarieties, Int. Math. Res. Not. IMRN, 2009, no. 19, 35953597.

[12] C. Laurent-Gengoux, A. Pichereau and P. Vanhaecke, Poisson structures, Grundlehren der Mathematischen Wissenschaften [Fundamental Principles of Mathematical Sciences], 347. Springer, Heidelberg, 2013.

[13] N. Lauritzen and J.F. Thomsen, Line bundles on Bott-Samelson varieties, J. Algebraic Geom. 13 (2004), 461-473.

[14] J.-H. Lu and Y. Mi, Generalized Bruhat cells and completeness of Hamiltonian flows of Kogan-Zelevinsky integrable systems, Lie Groups, Geometry, and Representation Theory. Progress in Mathematics, Birkhäuser, Cham, 326 (2018), 315-365.

[15] J.-H. Lu and V. Mouquin, Double Bruhat cells and symplectic groupoids, Transform. Groups, 23 (2018), no.3, $765-800$.

[16] J.-H. Lu and V. Mouquin, On the T-leaves of some Poisson structures related to products of flag varieties, Adv. Math., 306(2017), 1209-1261.

[17] J.-H. Lu and A. Weinstein, Poisson Lie groups, dressing transformations, and the Bruhat decomposition, J. Differential Geom., 31(1990), 501-526.

[18] J.-H. Lu and M. Yakimov, Group orbits and regular partitions of Poisson manifold, Comm. Math. Phys., 283 (2008), no.3, $729-748$.

[19] V.B. Mehta and A. Ramanathan, Frobenius splitting and cohomology vanishing for Schubert varieties, Ann. of Math., 122 (1) (1985), 27 - 40.

[20] J. Peng, Standard Poisson structure on Bott-Samelson varieties: degeneration and Frobenius splitting, PhD Thesis. Hong Kong: the University of Hong Kong, 2018.

[21] M. Semenov-Tian-Shansky, Dressing transformation and Poisson group actions, Publ. Res. Inst. Math. Sci., 21 (6) (1985), 1237 - 1260.

[22] J. Thomsen, Normality of crtain nilpotent varieties in positive characteristic, J. Algebra, 227 (2000), 595 613.

[23] A. Weinstein, The local structure of Poisson manifolds, J. Differential Geom., 18 (1983), 523-557.

[24] S. Yu, On the Knutson-Woo-Yong maps and some Poisson homogeneous spaces, PhD Thesis. Hong Kong: the University of Hong Kong, 2018.

Department of Mathematics, The University of Hong Kong, Pokfulam Road, Hong Kong

E-mail address: genesis@connect.hku.hk

Department of Mathematics, The University of Hong Kong, Pokfulam Road, Hong Kong

E-mail address: yusz@connect.hku.hk 